\title{
Guinea-Bissau: Second Review Under the Three-Year Arrangement Under the Extended Credit Facility and Financing Assurances Review-Staff Report; Informational Annex; Press Release on the Review
}

In the context of the second review under the three-year arrangement under the Extended Credit Facility and financing assurances review, the following documents have been released and are included in this package:

- $\quad$ Staff report for the Second Review Under the Three-Year Arrangement Under the Extended Credit Facility and Financing Assurances Review, prepared by a staff team of the IMF, following discussions that ended on March 23, 2011, with the officials of Guinea-Bissau on economic developments and policies. Based on information available at the time of these discussions, the staff report was completed on May 10, 2011. The views expressed in the staff report are those of the staff team and do not necessarily reflect the views of the Executive Board of the IMF.

- Informational annex

- $\quad$ Press release on the completion of the review

The policy of publication of staff reports and other documents allows for the deletion of market-sensitive information.

\author{
Copies of this report are available to the public from \\ International Monetary Fund • Publication Services \\ $70019^{\text {th }}$ Street, N.W. • Washington, D.C. 20431 \\ Telephone: (202) 623-7430 • Telefax: (202) 623-7201 \\ E-mail: publications@imf.org Internet: http://www.imf.org
}

\section{International Monetary Fund Washington, D.C.}


INTERNATIONAL MONETARY FUND

GUINEA-BISSAU

\section{Second Review Under the Three-Year Arrangement Under the Extended Credit Facility and Financing Assurances Review}

Prepared by the African Department

(In consultation with other departments)

Approved by Roger Nord and Thomas Dorsey

May 10, 2011

\section{Executive Summary}

On December 13, 2010, the Executive Board concluded the first review of the ECF arrangement and Guinea-Bissau reached the completion point under the Enhanced HIPC Initiative.

Economic growth is expected to accelerate moderately to 4.3 percent this year, driven by rising cashew production, higher cashew prices, and rebuilding of infrastructure.

Inflation is expected to rise to about 4 percent this year, driven by higher import prices for fuel and food, before coming back within the WAEMU target range in 2012.

Performance under the ECF-supported program has been satisfactory. The authorities have met all performance criteria through end-December 2010 and all structural benchmarks for the second review.

The program continues to be fully financed. In addition to budget support expected under the program, the government received a new grant from Angola largely allocated to restore the public investment program.

Following the HIPC completion point, the momentum of economic reforms has gained pace. The government is implementing measures to strengthen revenue collection and improve public financial management and is moving ahead with structural reforms for the third and fourth review.

Staff recommends completion of the second review of the ECF arrangement. The authorities' satisfactory performance under challenging circumstances and their commitment to successful implementation of the program warrant support from the IMF. Completion of the review will enable the disbursement of SDR 2.414 million (17 percent of quota).

Discussions were held on March 10-23, 2011, in Bissau. The team comprised Mr. Drummond (head), Mr. Daal, Mr. Peña (all AFR), Mr. Jewell (SPR), and Mr. Fonseca (local economist). Mr. Torrez, expected to be the new resident representative as of June, was introduced to the authorities. Mr. Tall, from the Executive Director's office, assisted the authorities during the discussions. 
I. Satisfactory macroeconomic and Program Performance...................................................

II. The Economic Outlook Remains Favorable ………….................................................

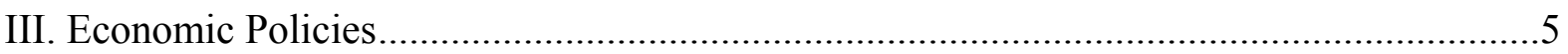

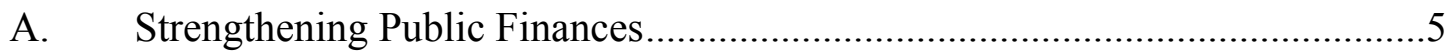

B. Modernizing the Public Administration.........................................................

C. Reducing Poverty ...................................................................................

D. Managing Debt and Financing Assurances.........................................................

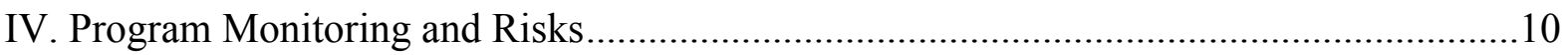

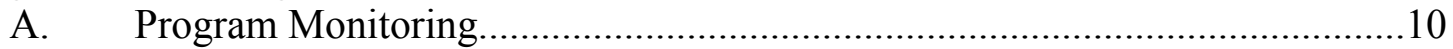

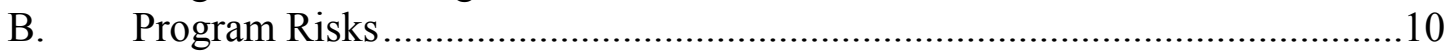

V. Statistical Issues and Capacity Development..................................................................

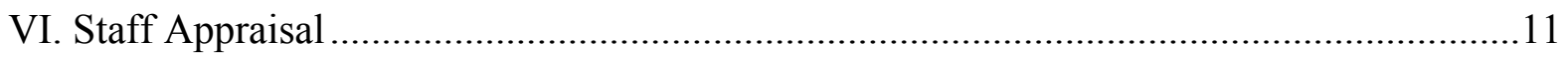

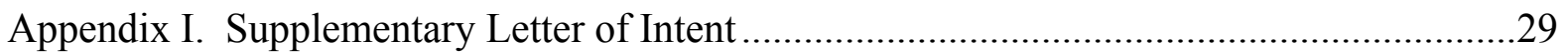

Attachment I. Supplementary Memorandum of Economic and Financial Policies for 2011..30

Attachment II. Technical Memorandum of Understanding ...................................................42

Figures

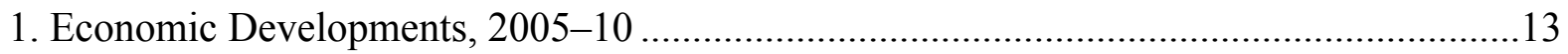

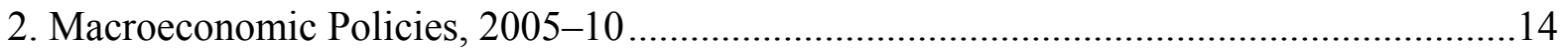

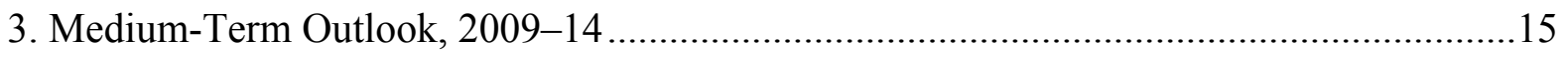

4. Impact of Food and Fuel Prices on Inflation ………...................................................16

Boxes

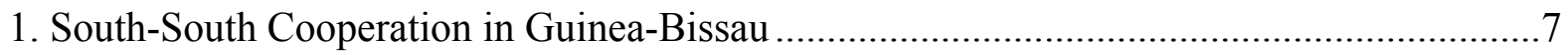

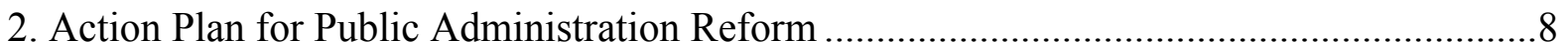

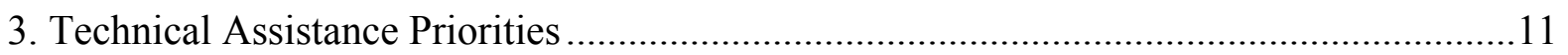

Tables

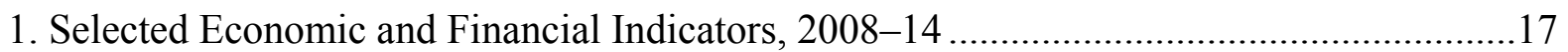

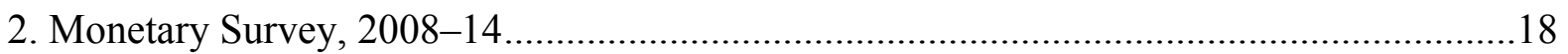

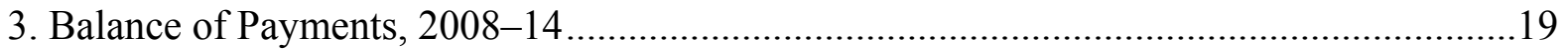

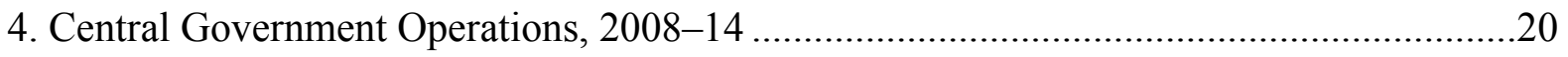

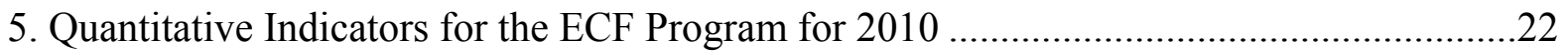

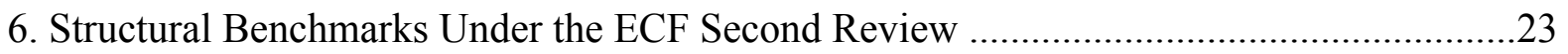

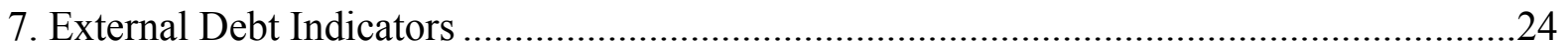

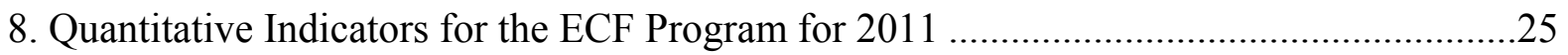

9. Structural Benchmarks under the ECF Third and Fourth Review ....................................26

10. Reviews and Disbursements Under the Three-Year ECF Arrangement, 2010-13 ...........27

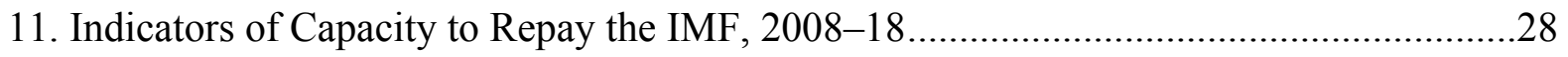




\section{AbBreviations}

\begin{tabular}{ll} 
AfDB & African Development Bank \\
AFRITAC & African Regional Technical Assistance Center \\
ASYCUDA & Automated Systems for Customs Data \\
BCEAO & Central Bank of West African States \\
ECF & Extended Credit Facility \\
EPCA & Emergency Postconflict Assistance \\
EU & European Union \\
HIPC & Highly Indebted Poor Countries \\
MDG & Millennium Development Goals \\
MDRI & Multilateral Debt Relief Initiative \\
MEFP & Memorandum of Economic and Financial Policies \\
PC & performance criterion \\
PFM & public financial management \\
PIP & Public Investment Program \\
PRGF & Poverty Reduction and Growth Facility \\
PRS & poverty reduction strategy \\
PRSP & Poverty Reduction Strategy Paper \\
SIGFIP & Integrated Public Finance Management System \\
SIGRHAP & Personnel Management System \\
SYGADE & Debt Management System \\
TMU & Technical Memorandum of Understanding \\
UNCTAD & United Nations Conference on Trade and Development \\
VAT & value-added tax \\
WAEMU & West African Economic and Monetary Union \\
WEO & World Economic Outlook \\
& \\
\hline
\end{tabular}




\section{Satisfactory macroeconomic and Program Performance}

1. Economic growth has been resilient, owing to a rebound in the price of cashew exports and improved liquidity in the banking system. Growth accelerated slightly to 3.5 percent in 2010, as strong terms-of-trade gains more than offset a weather-related decline in the cashew harvest (Figure 1, Table 1). Exports were supported by rising credit, as liquidity in domestic commercial banks improved, reflecting the government's arrears clearance early last year (Table 2). Imports, however, were well contained and the external current account deficit, excluding official transfers, narrowed by about 4 percentage points of GDP (Table 3). Inflation picked up slightly at the end of the year on the back of rising import prices for food and fuel, but, at 1.1 percent, remained below the WAEMU convergence criterion.

2. Fiscal performance remained on track. Revenue collection was stronger than expected, reflecting sustained economic activity and tighter controls at customs and revenue administration. Expenditures were contained below program, reflecting lower outlays in goods and services and underexecution of the public investment program due to shortfalls in budget support (Figure 2, Table 4).

\section{Performance under the ECF-supported program has been satisfactory. All} performance criteria and quantitative targets for end-December, including the indicative targets for social and priority spending, and all the structural benchmarks for the second review have been met (Tables 5 and 6). The authorities made the link from the treasury to customs administration operational; approved the action plan for public administration reform; and launched the unified payroll system at the ministries of finance and labor and public administration.

\section{The ECONOMic OUTLOOK REMAins FAVORABLE}

\section{The authorities' medium-term macroeconomic objectives under the ECF-} supported program remain unchanged (IMF Country Report No. 10/379 and Figure 3). The authorities reiterated their commitment to (i) raise real growth to $4 \frac{1}{2}$ percent by program end, (ii) maintain low annual inflation, and (iii) keep the budget deficit consistent with available financing.

5. The growth outlook for 2011 is favorable, but inflation and balance of payments pressures reflect higher import prices for food and fuel.

- $\quad$ Economic growth is expected to accelerate moderately to 4.3 percent, driven by rising cashew production, higher cashew prices, and a steady rebuilding of infrastructure, especially roads, electricity, and water.

- Inflationary and balance of payment pressures reflect rising import prices for food and fuel. The pass-through of imported prices to domestic prices in Bissau is high due to a high dependence on imports and limited supply response (Figure 4). Headline 
inflation is now projected at 4 percent, above the WAEMU convergence criterion. However, core inflation is expected to remain subdued, and headline inflation should return to the WAEMU target range in 2012, once the one-off effect of rising import prices subsides. Driven by higher import prices, the external current account deficit (excluding official transfers) is expected to widen slightly.

6. The authorities agreed that adjustment to the food and fuel price shock would be preferable to accommodation. A full pass-through of price changes over time should encourage consumers and producers to adjust. Staff and the authorities shared the view that general price subsidies would not benefit the poor and, in the absence of well-targeted programs and the time it takes for these to be developed, the government would ensure that resources for social and other priority spending are fully allocated as planned under the program.

7. Budget support is projected to be larger than expected under the program. In addition to the budget support expected under the program (from the World Bank and the African Development Bank), the government received a new grant from Angola (1.3 percent of GDP) (see Box 1). The grant will allow the government to restore its public investment program and repay previous years' arrears to the private sector ( 0.4 percent of GDP). Consultations with the EU under Article 96 of the Cotonou Agreement took place in Brussels on March 29. The EU announced it will condition its support to Guinea-Bissau on the implementation of undertakings by the authorities in several areas, including security sector reform and the appointment of military personnel. The program continues to assume no financial support from the EU this year.

\section{ECONOMic Policies}

8. The discussions were marked by general agreement on the need to maintain fiscal discipline and sustain the post-HIPC reform momentum, with a focus on mobilizing more revenue, modernizing the public administration, and managing debt. Economic policies remain broadly unchanged as expressed in the authorities' Supplementary Letter of Intent (Appendix I) and the attached Supplementary Memorandum of Economic and Financial Policies and the Technical Memorandum of Understanding, which update and supplement those of November 16, 2010.

\section{A. Strengthening Public Finances}

\section{Fiscal Policy}

9. The 2011 budget approved by Parliament is consistent with the medium-term objectives of the government. The budget includes important measures to strengthen fiscal revenue, including by reducing implicit import subsidies, raising the customs reference price for cashew exports, and increasing the tax rate on advance payment of profit tax (ACI) from 3 to 5 percent for the informal sector. On the spending side, the budget preserves social and 
other priority outlays and freezes wages while incorporating contractual workers in the health and education sector in the wage bill.

10. The fiscal program continues to be fully financed (Text table). Revenues are projected to increase as planned under the program, with the overperformance in 2010 (about $1 / 2$ percentage points of GDP) carrying over to 2011. This offsets higher-than-budgeted spending due to a harmonization of wages across defense and security forces and upwardly revised wage estimates for health and education contractual workers. At the same time, the public investment program-which was affected in the past by lack of resources and delayed budget support disbursements-is being restored with the assistance from Angola. As a result, domestic financing of the budget remains unchanged. The financing gap is

\begin{tabular}{lrrr} 
& \multicolumn{3}{c}{ Text Table. Guinea-Bissau: Fiscal Developments $2010-11$} \\
\hline & 2010 & \multicolumn{2}{c}{2011} \\
\cline { 3 - 5 } & Est. & Prog. & Proj. \\
\hline & \multicolumn{3}{c}{ Percent of GDP } \\
Revenue and Grants & 20.4 & 18.2 & 19.8 \\
Revenue & 10.8 & 10.7 & 11.1 \\
Grants & 9.7 & 7.5 & 8.7 \\
$\quad$ Budget support & 2.3 & 1.7 & 3.0 \\
Total Expenditure & 20.7 & 20.6 & 21.8 \\
Current expenditure & 11.9 & 11.9 & 12.4 \\
Capital expenditure & 8.8 & 8.6 & 9.5 \\
Net domestic arrears and float & -2.3 & -0.4 & -0.8 \\
Overall balance, including grants (cash) & -2.5 & -2.8 & -2.8 \\
Financing & 2.5 & 2.8 & 2.8 \\
Domestic financing & 1.8 & 0.0 & 0.0 \\
Foreign financing & 0.8 & 2.0 & 2.0 \\
Gross financing gap & 0.0 & 0.8 & 0.8 \\
\hline Sources: Guinea-Bissau authorities and IMF staff estimates and projections. & &
\end{tabular}
expected to be filled by the ECF arrangement.

\section{Mobilizing more revenues}

\section{The government is working to further broaden the tax base and improve capacity and efficiency in customs and revenue administration.}

- On tax administration, the government prepared an action plan, with technical assistance from AFRITAC West, to: (i) revise taxpayer segmentation based on turnover criteria, with thresholds for small and large taxpayers at CFAF10 and 40 million, respectively; (ii) cross-check ASYCUDA data with tax returns for the 300 largest importers in 2009-10; and (iii) audit importers underreporting tax liabilities, beginning with largest gaps.

- $\quad$ On customs, the government will (i) finish customizing and testing MEFP $q 16$ the ASYCUDA ++ system with the parameters for Guinea-Bissau and train staff with the help of UNCTAD; and (ii) finish the comprehensive review of all custom exemptions and submit a report to the Council of Ministers by June.

\section{The government is reinforcing fiscal revenues this year and next by raising} customs reference prices for identified imports, thus gradually eliminating import subsidies. By end March, the government eliminated the reference price of sugar for customs purposes and raised the reference price for diesel. In addition, it will:

(i) gradually adjust the reference price for imported diesel toward market

MEFP $q 12$ levels by end-June; (ii) adjust domestic fuel prices monthly, beginning in June, to allow for a full pass-through of international prices into domestic prices; and (iii) review the reference price of certain imports (rice, flour, diesel) by year-end, with the goal of raising them to 
market levels as part of the 2012 budget; and (iv) reduce or eliminate customs exemptions to be identified by the government's current review. On an annual basis, these measures could yield about $1 / 2$ to 1 percent of GDP.

\section{Box 1. South-South Cooperation in Guinea-Bissau}

Traditionally, the main development partners of Guinea-Bissau have been the European Union (EU), European bilateral donors, and multilateral organizations such as the World Bank, the African Development Bank, the United Nations, and the Economic Community of West African States (ECOWAS). During 2000-2009, among donors that report to the OECD Development Assistance Committee (DAC), the EU (US\$294 million), Portugal (US\$132 million), the World Bank (US\$125 million), Italy (US\$78 million), and Spain (US\$55 million) provided the most development assistance to Guinea-Bissau. Not captured in these figures, however, is development assistance from key providers of South-South cooperation.

Providers of South-South cooperation in Guinea-Bissau have typically provided project financing, often for infrastructure, as well as technical assistance. Southern providers include Angola, Brazil, and China.

Angola provided a US\$12-million (about 1.3 percent of GDP) grant in February 2011, which the authorities intend to use to finance roads and agriculture projects and to pay previous years' arrears to the private sector. In October 2010, Angola announced that it would open a US\$25-million line of credit to support entrepreneurs from both countries who want to invest in Guinea-Bissau. In 2008, Angola provided US\$10 million in budget support. In addition to financial assistance, Angola has been actively involved in security reform.

Brazil has cooperated with Guinea-Bissau across several sectors. It has provided technical assistance to increase agricultural production; established training centers for the military, the police, teachers, and ex-combatants; and helped build capacity to combat HIV/AIDS. The United Nations Development Program (UNDP) estimates that Brazil's bilateral assistance to Guinea-Bissau totaled US\$6.2 million during 2006-2009.

China has realized several large projects in Bissau, including a 20,000-seat stadium, the National Assembly building (US\$6 million), a new government office (US\$12 million) that will house 12 ministries, and a hospital (US\$8 million). China has also provided technical assistance to improve rice production. 


\section{B. Modernizing the Public Administration}

13. To improve the efficiency of the civil service, the government approved in March an action plan for the reform of the public administration. The plan aims to reduce the civil service and upgrade the qualifications and working MEFP q17 conditions for civil servants (see Box 2). While the reform is gradual and figures will be finalized only after the unification of the payroll system, the plan is to start bringing the ratio of employees in line with the norm in the WAEMU region.

\section{The government began using the unified payroll system in most ministries in}

March. Next steps will be to extend the payroll system to all ministries and start using the personnel management system (SIGRHAP), being implemented with the support of the EU, this fall. This would allow the government to better MEFP $\mid 18$ control, record, and manage the payroll.

\section{Box 2. Action Plan for Public Administration Reform}

Modernizing the public administration through civil service and security reforms has been at the center of the reform agenda in Guinea-Bissau for many years. The comprehensive biometric census completed by the government in June last year gave an accurate account of all employees working at the public administration and helped lay the groundwork for the government to prepare a medium-term action plan for reforming the public administration. The timetable for its implementation covers 2011-15. The plan was prepared by the Ministry of Labor and Public Administration with the assistance of the EU Public Administration Reform Program (PARAP).

In March, the government approved its plan for public administration reform.

- Key objectives are: to reduce the size of the civil service, with a view to start bringing the ratio of employees from 10 to 6-7 workers per 1,000 inhabitants, in line with the norm in the WAEMU region; to upgrade the qualifications and working conditions for civil servants; and, to increase the efficiency and quality of public administration.

- Measures identified under the plan include: (i) retiring all employees who exceed the mandatory age; (ii) reinserting the group of civil servants who are in an excess pool of workers ("redundant workers") into the private sector; (iii) providing training for civil servants who will remain in the public administration and for employees reinserting into the private sector; and (iv) revising the salary scale.

\section{The government is coordinating with partners to implement the defense and} security sector reform. The government approved in March the road map for the reform, designed in coordination with ECOWAS and the Community of Portuguese Language Countries (CPLP). The reform is being supported by Angola, which has stepped up its 
involvement by providing training and improving living conditions and infrastructure for the police and military forces. Civilian-led aspects of the reform are being supported by the UN under its Priority Plan for Guinea-Bissau approved in February, with an allocation of US\$16 million.

\section{Reducing Poverty}

16. The government is finalizing its new five-year poverty reduction strategy (PRS). To ensure implementation, the government will better coordinate with donors and development partners. Priorities are to identify economic projects in line with the PRS, fully implement the public investment MEFP q14 program (PIP), and preserve spending on the priority areas identified by the government: health, education, agriculture, and infrastructure.

\section{Managing Debt and Financing Assurances}

17. The results of the low-income country debt sustainability analysis (DSA) indicate that Guinea-Bissau faces a moderate risk of debt distress. ${ }^{1}$ This assumes the full delivery of HIPC assistance, additional bilateral assistance from the Paris Club beyond HIPC, topping up, and debt relief under the Multilateral Debt Relief Initiative. Although key debt indicators are expected to remain below their relevant policy-dependent thresholds (Table 7), stress tests reveal that Guinea-Bissau is vulnerable to shocks, particularly to exports.

\section{The government is taking steps to implement the HIPC completion point.}

Following the completion point, the government sent letters to all external creditors asking them to confirm their intention to provide HIPC and topping-up debt relief. Portugal, the largest non-Paris Club creditor, and Algeria have MEFP $\uparrow 25$ agreed in principle to forgive 100 percent of their claims. Negotiations with Angola are ongoing, and the authorities are evaluating restructuring proposals from Saudi Arabia and Kuwait. The Islamic Development Bank and Caixa Geral de Depósitos have both indicated their willingness to restructure their claims. The latter is Guinea Bissau's lone commercial creditor; all the debt is in arrears and is eligible to be restructured under HIPC. A Paris Club meeting to conclude a new agreement implementing HIPC completion point has been scheduled for May.

19. The government has prepared an action plan to strengthen debt management. It plans to hire and train new staff, produce quarterly electronic reports on public debt by June 2011, and use the debt management system (SYGADE) by December to record, monitor, and manage all public debt, MEFP q21 with assistance from the United Nations Conference on Trade and Development (UNCTAD).

\footnotetext{
${ }^{1}$ IMF Country Report No. 10/380.
} 
20. The government has requested to renegotiate debt owed to the Central Bank of West African States (BCEAO). The debts to the BCEAO (about CFAF90 billion or 20 percent of GDP) consist of a required capital contribution to join the WAEMU and other obligations arising from previous rescheduling MEFP q22 agreements.

21. The government will continue with its medium-to-long-term domestic arrears clearance strategy. The government will continue to pay the pre-1999 audited and verified arrears (3.3 percent of GDP as of end-2010). A team of independent auditors issued a preliminary audit report for the arrears MEFP q23 of 2000-07, estimated at about 18.4 percent of GDP. The authorities are verifying the audit and plan to register at the Treasury and incorporate verified arrears into their medium-tolong-term arrears strategy by making annual payments consistent with available resources.

\section{Program Monitoring AND Risks}

\section{A. Program Monitoring}

22. All performance criteria and structural benchmarks under the program for 2011 remain unchanged (Tables 8 and 9). The indicative targets for revenue, social and other priority spending, and domestic primary deficit were adjusted in line with revised fiscal projections for 2011 (Table 8). The third review is expected in December 2011 and the fourth review in May 2012 (Table 10).

23. The definition of external debt in the Technical Memorandum of Understanding has been changed from a residency to a currency basis, in line with IMF efforts to harmonize the definition of external debt among countries in the WAEMU area.

\section{B. Program Risks}

24. Guinea-Bissau faces significant risks, but risks to the IMF's resources are moderate. The main concerns are political instability, vulnerability to external shocks, and the possibility of inadequate donor support, including delays in donor disbursements. The country's capacity to repay the IMF is reasonably sound (Table 11). Risks to IMF resources are mitigated to a significant degree by the BCEAO's role as fiscal agent and its solid track record in making payments to the IMF, even without reimbursement by Guinea-Bissau.

\section{Statistical Issues AND CAPACITy DEVElopMent}

25. The authorities agreed on the need for better data dissemination and for capacity building to ensure effectiveness of fiscal reforms. The authorities will publish preliminary national account data for 2010, based on the SNA93, this year. They will continue to publish a WAEMU harmonized consumer price index. The IMF is providing technical support in public financial management, revenue administration, and national accounts statistics (see Box 3). 


\section{Box 3. Technical Assistance Priorities}

The following priorities have been identified by the authorities:

Revenue administration: The goal is to strengthen customs clearance and the internal tax collection unit. In 2010, a technical assistance mission from the Fiscal Affairs Department made recommendations in these areas, and the government has incorporated them in their economic program.

Tax policy: The short-term goals are to broaden the tax base, including by closing tax loopholes, and to consolidate and rationalize tax exemptions. A medium-term goal is to implement a VAT system.

Debt management: Following HIPC completion point, the goal is to strengthen the unit of public debt at the Ministry of Finance to record, manage, and produce quarterly reports on public debt.

Public expenditure and financial management: The aim is to enhance budget preparation and control in budget execution; to strengthen accounting, recording, and reporting procedures; and to reinforce internal and external controls.

Statistics: The authorities are working to strengthen national accounts statistics and to start preparing national accounts based on SNA98.

\section{StAFF APPRAisal}

26. Staff welcomes the authorities' satisfactory performance under the ECFsupported program. The government maintained macroeconomic stability despite challenging circumstances and a large shortfall in budget support last year.

27. The government has maintained the momentum of economic reforms. It has taken steps to mobilize more revenue and better manage public finances. It will be important for the government to follow through with its plans to modernize the public administration and revamp its personnel management system.

28. Staff supports the authorities' decision to fully pass through global food and fuel price increases into domestic prices and to eliminate import subsidies. Bringing import prices for custom purposes closer to market levels will encourage consumers and producers to adjust over time and should help create much needed fiscal space for social and other priority spending. 
29. Implementing HIPC debt relief and a prudent debt management strategy will be critical for securing debt sustainability. The priority is for the government to pursue debt restructuring agreements with all its external creditors. Developing a prudent debt management strategy and strengthening the debt unit with support from development partners are imperatives in the wake of the HIPC completion point.

30. Staff recommends the completion of the second review under the ECF arrangement and the financing assurances review, and supports the authorities' request for the third disbursement under the three-year ECF arrangement. The authorities' satisfactory performance under challenging circumstances, their efforts to move ahead with economic reforms, and their commitment to successful implementation of the program, as expressed in their Letter of Intent (Appendix I), warrant support from the IMF. 
Figure 1. Guinea-Bissau: Economic Developments, 2005-10

GDP growth accelerated in 2010, fueled by ...

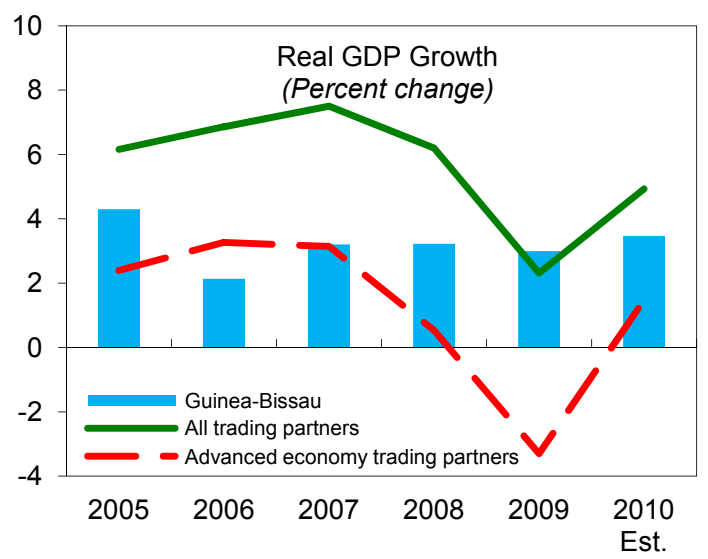

Rising cashew export receipts...

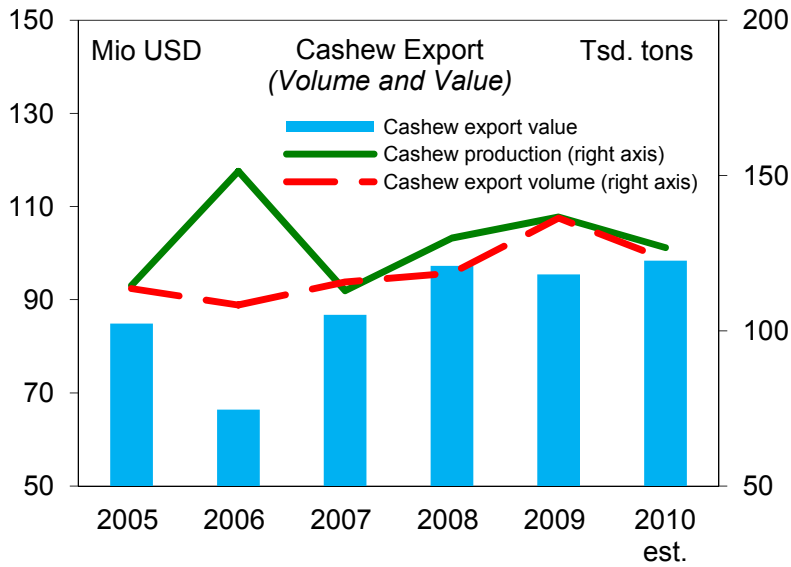

...contributed to narrowing the external current account deficit.

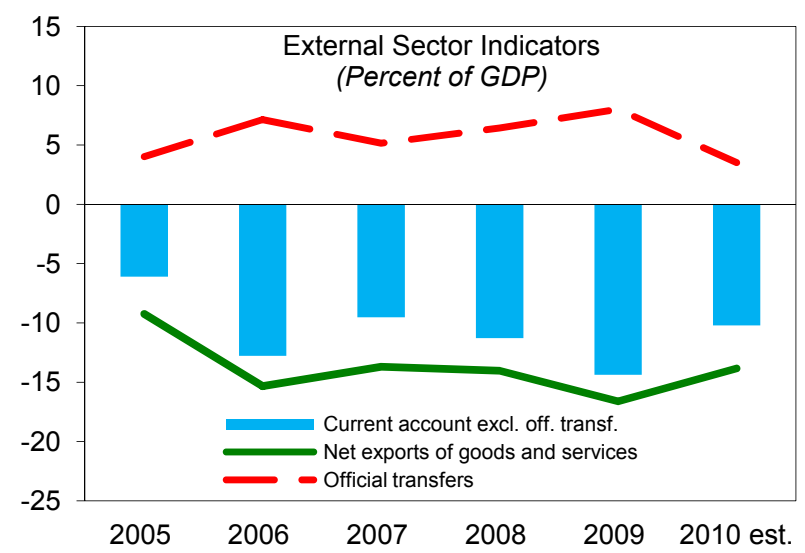

... terms of trade gains owing to higher cashew prices.

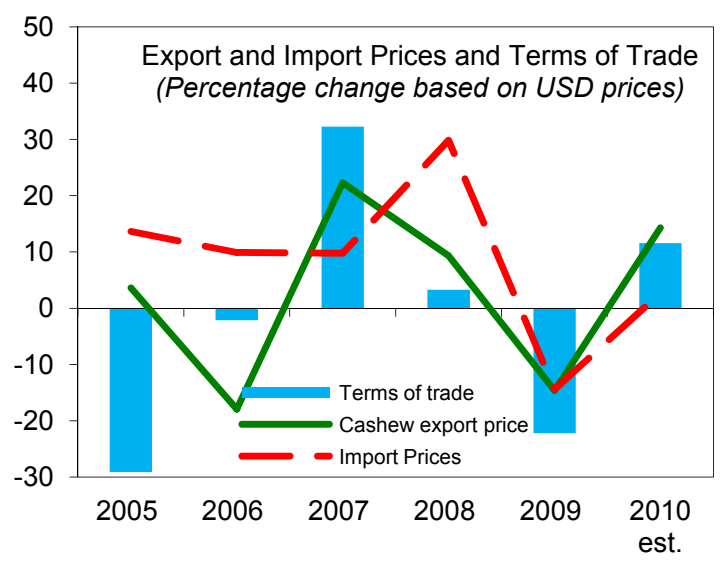

... supported growth in disposable income and domestic demand and

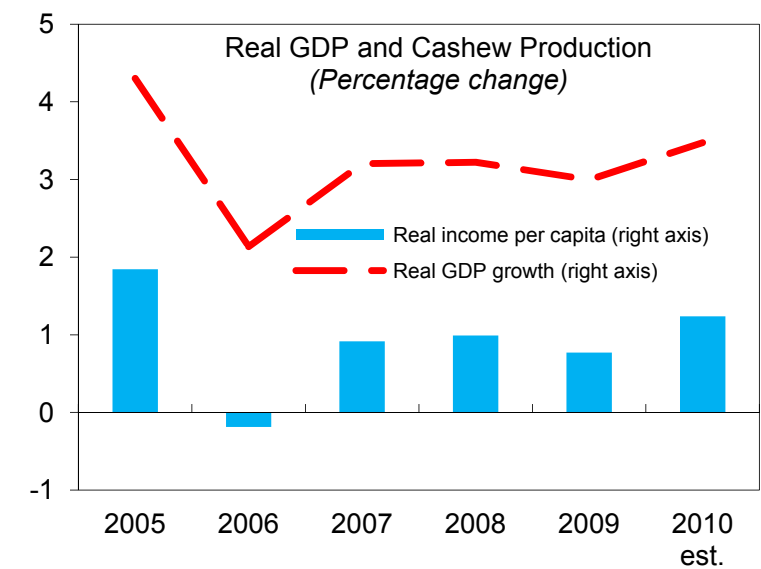

Inflation accelerated but remained within the WAEMU target.

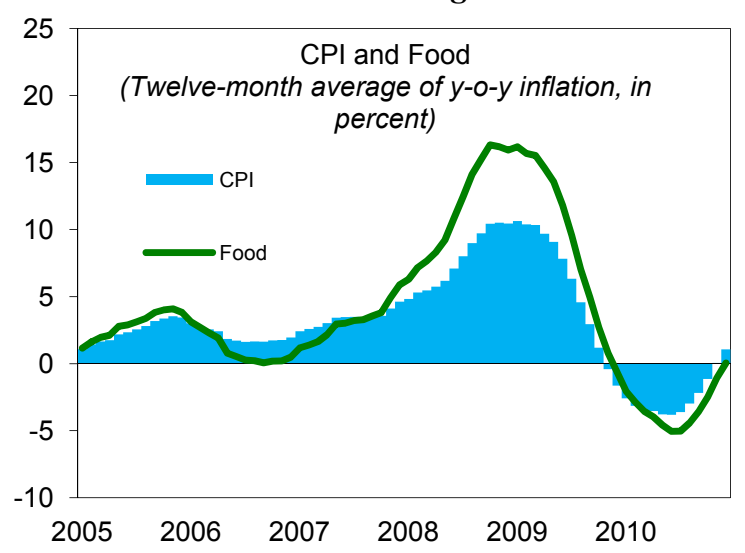

Sources: Guinea-Bissau authorities and IMF staff estimates. 
Figure 2. Guinea-Bissau: Macroeconomic Policies, 2005-10

Fiscal performance was satisfactory,...

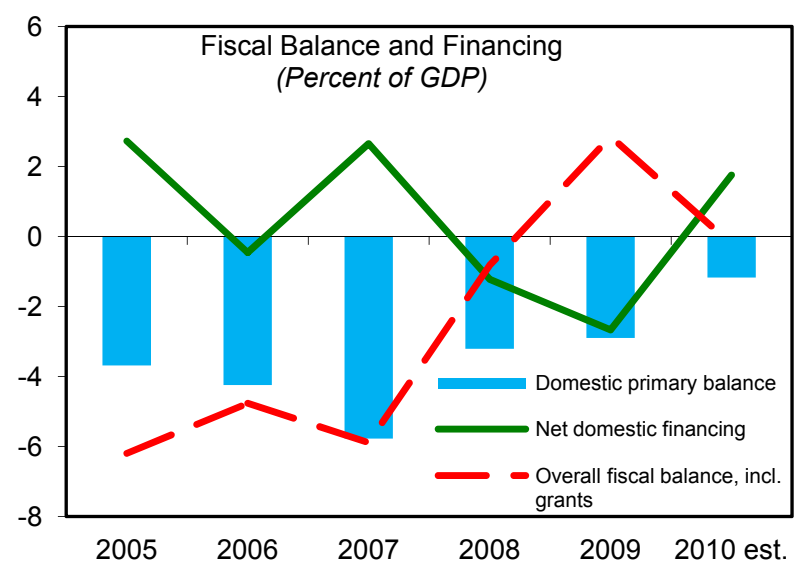

... and the government contained current spending.

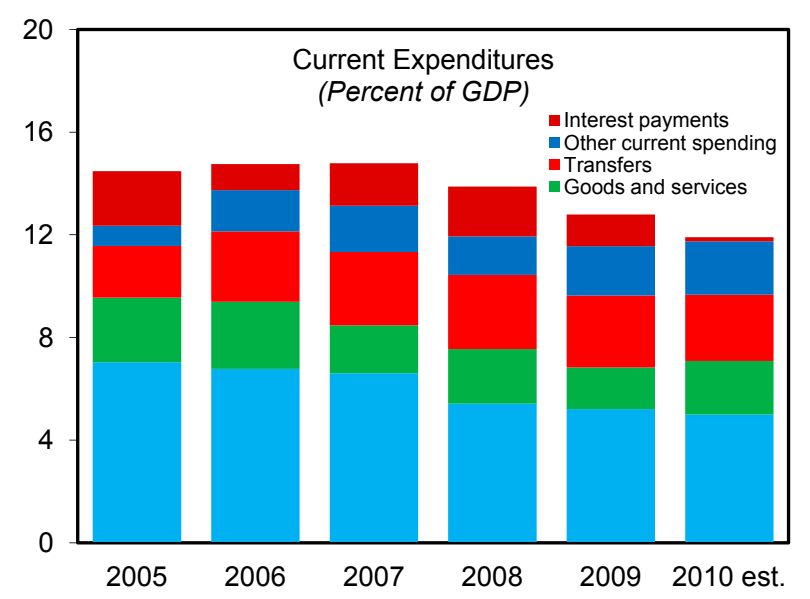

... and private sector credit growth rebounded.

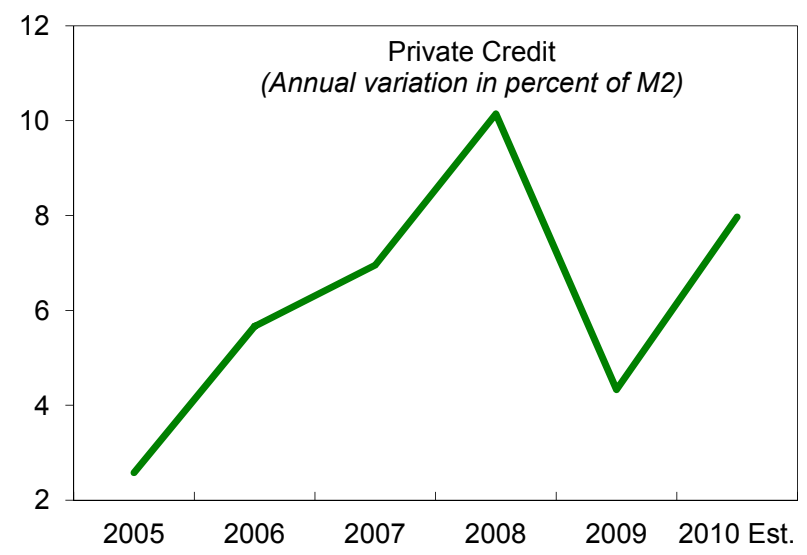

...with higher-than-programmed revenue partly compensating for shortfalls in budget support, ...

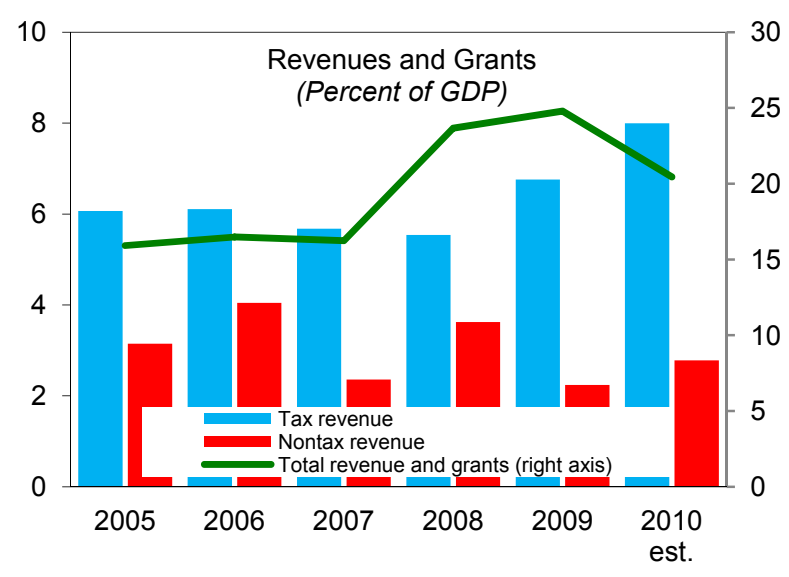

The WAEMU monetary policy stance was accommodating, ...

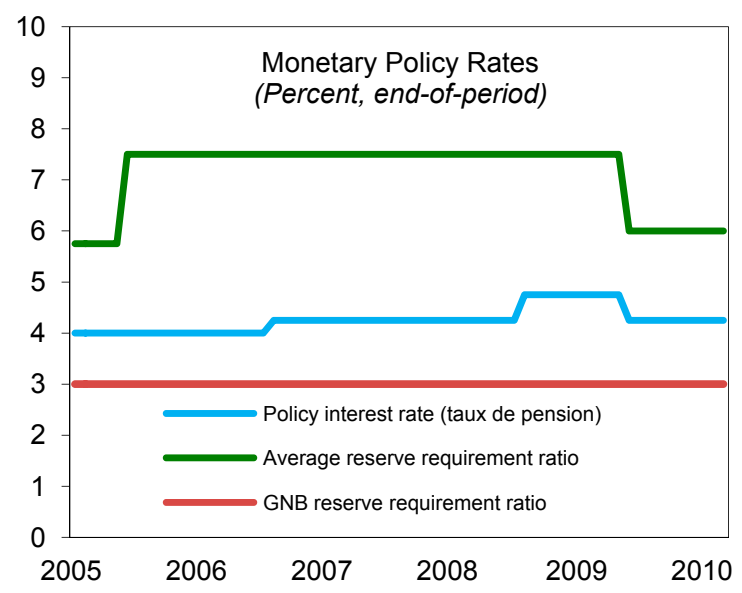

Debt levels fell substantially with debt relief.

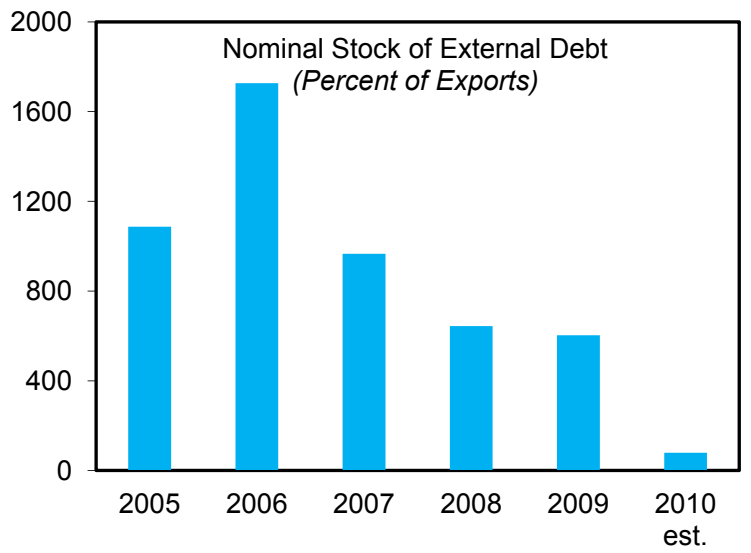

Sources: African Department database, BCEAO, Guinea-Bissau authorities, and IMF staff estimates. 
Figure 3. Guinea-Bissau: Medium-Term Outlook, 2009-14

A modest pick-up in growth is expected, ...

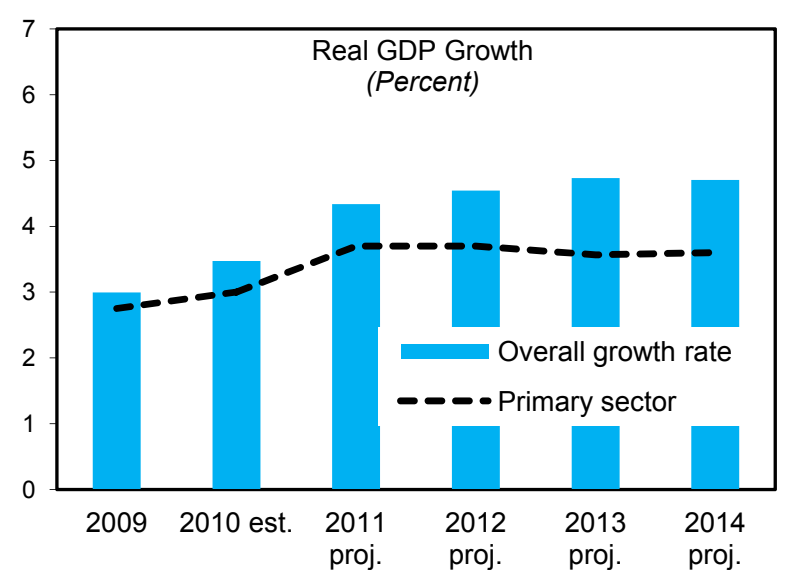

The domestic primary deficit will be contained in line with available financing ...

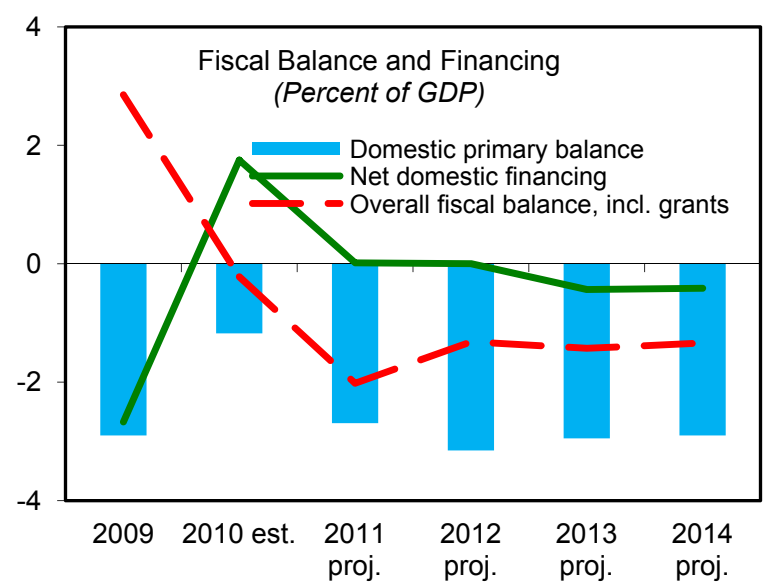

... increased revenue collection.

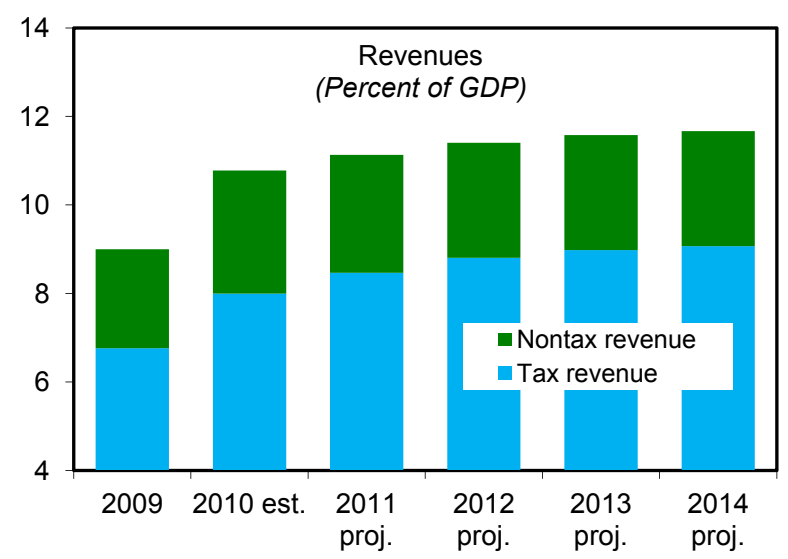

... while inflation is expected to rise temporarily in 2011.

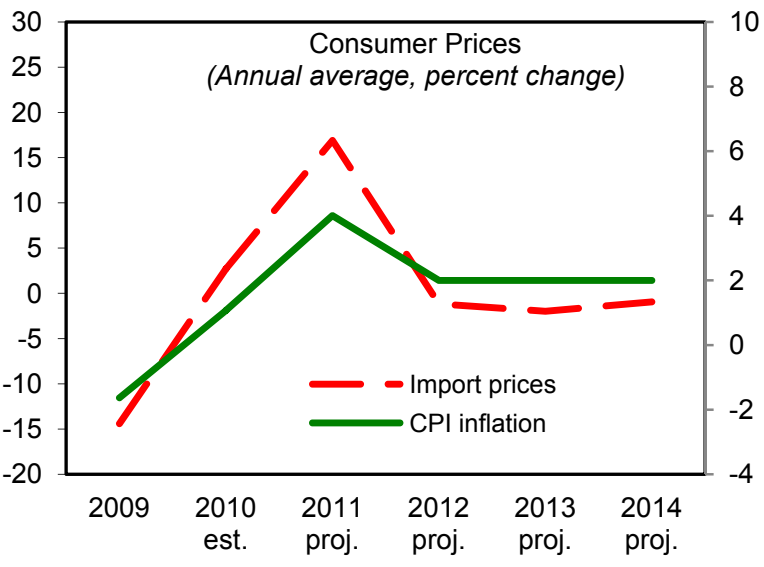

... through expenditure controls, debt relief, and...

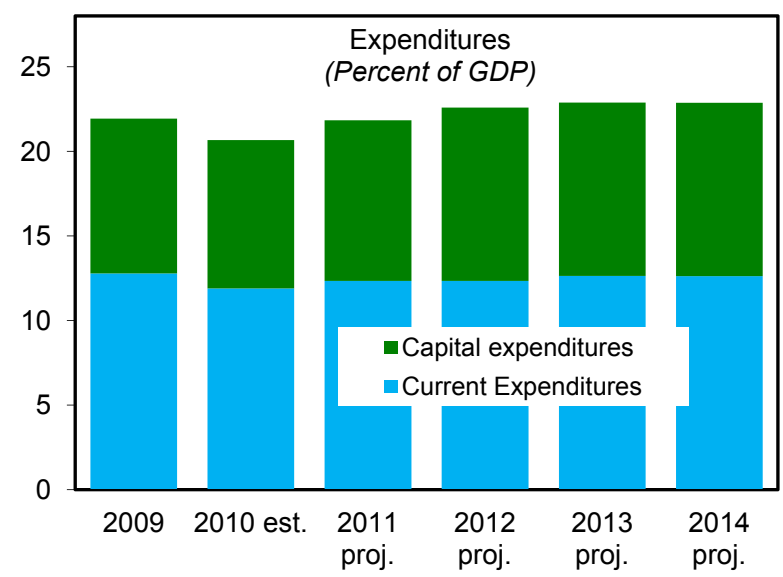

The external current account deficit is expected to be contained, in line with net exports.

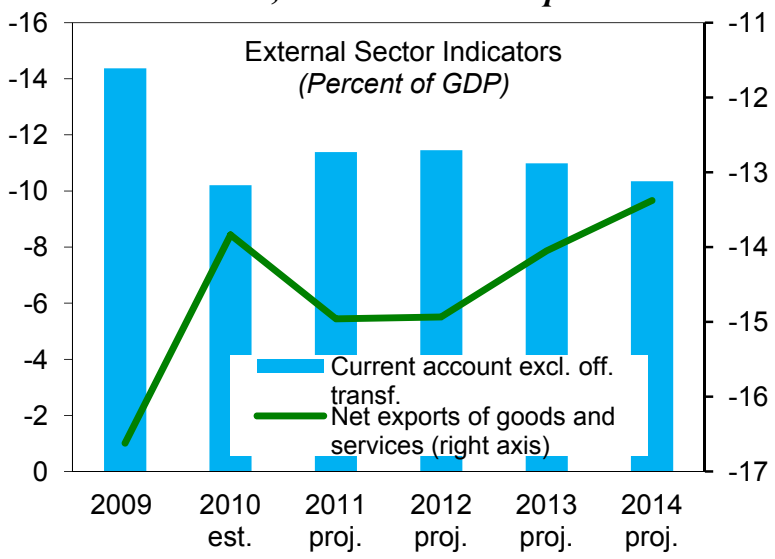

Sources: Guinea-Bissau authorities and IMF staff estimates and projections. 
Figure 4. Guinea-Bissau: Impact of Food and Fuel Prices on Inflation
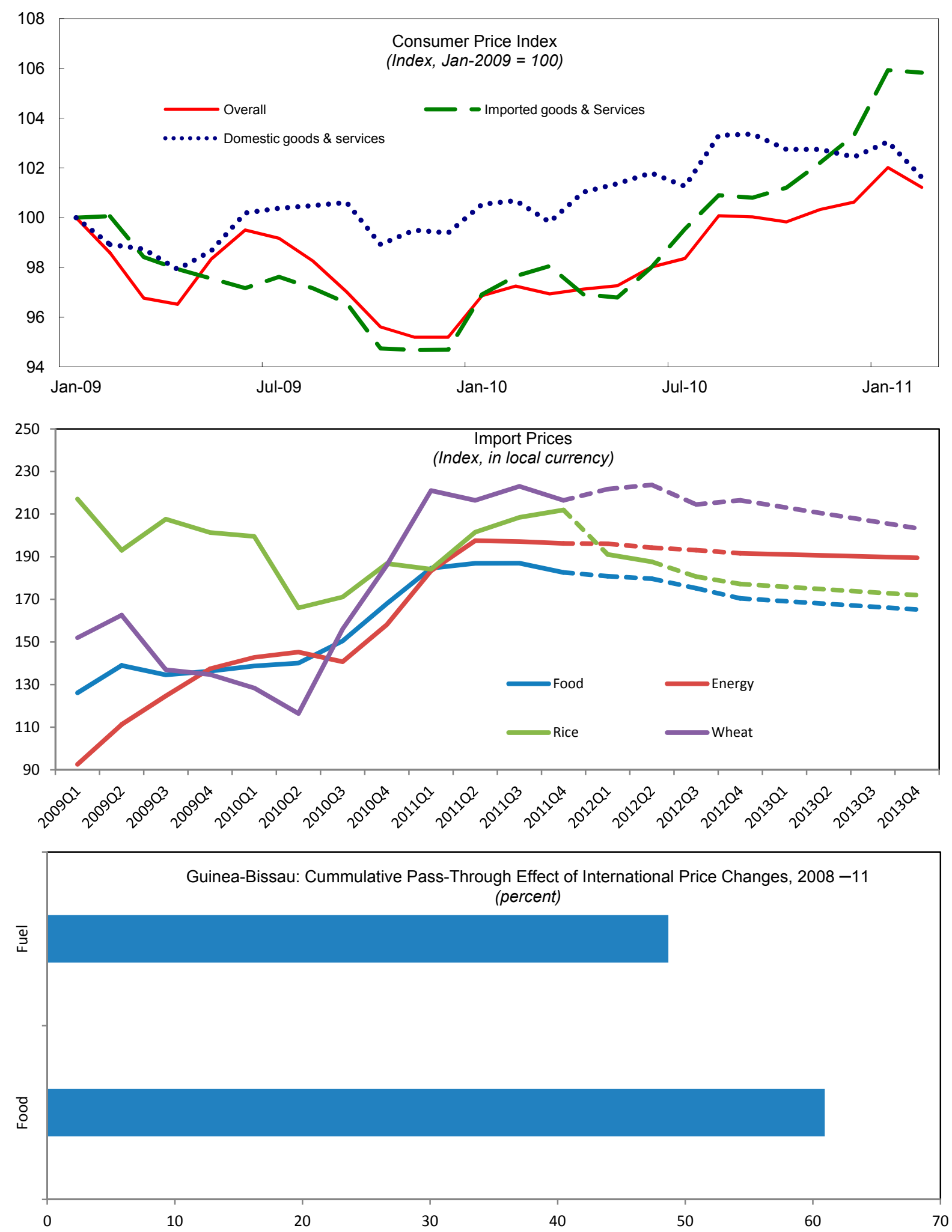

Sources: Guinea-Bissau authorities and IMF staff estimates and projections. 
Table 1. Guinea-Bissau: Selected Economic and Financial Indicators, 2008-14

\begin{tabular}{|c|c|c|c|c|c|c|c|c|c|}
\hline & \multirow[t]{2}{*}{2008} & \multirow[t]{2}{*}{2009} & \multicolumn{2}{|c|}{2010} & \multicolumn{2}{|c|}{2011} & \multirow{2}{*}{$\begin{array}{l}2012 \\
\text { Proj. }\end{array}$} & \multirow{2}{*}{$\begin{array}{l}2013 \\
\text { Proj. }\end{array}$} & \multirow{2}{*}{$\begin{array}{l}2014 \\
\text { Proj. }\end{array}$} \\
\hline & & & Prog. ${ }^{1}$ & Est. & Prog. ${ }^{2}$ & Proj. & & & \\
\hline & \multicolumn{9}{|c|}{ (Annual percentage change, unless otherwise indicated) } \\
\hline \multicolumn{10}{|l|}{ National accounts and prices } \\
\hline Real GDP at market prices & 3.2 & 3.0 & 3.5 & 3.5 & 4.3 & 4.3 & 4.5 & 4.7 & 4.7 \\
\hline Real GDP per capita & 1.0 & 0.8 & 0.5 & 1.2 & 2.1 & 2.1 & 2.3 & 2.5 & 2.4 \\
\hline GDP deflator & 10.5 & 1.1 & 2.4 & 1.7 & 2.1 & 3.8 & 1.8 & 2.2 & 1.9 \\
\hline Consumer price index (annual average) & 10.4 & -1.6 & 2.5 & 1.1 & 2.5 & 4.0 & 2.0 & 2.0 & 2.0 \\
\hline \multicolumn{10}{|l|}{ External sector } \\
\hline Exports, f.o.b. (based on US\$ values) & 13.1 & -3.6 & 13.6 & 2.1 & 11.2 & 16.4 & 0.6 & 2.9 & 2.8 \\
\hline Imports, f.o.b. (based on US\$ values) & 18.8 & 1.5 & 9.2 & -2.9 & 6.0 & 19.9 & 2.7 & 0.5 & 0.9 \\
\hline Export volume & 3.4 & 12.9 & 4.3 & -10.6 & 7.4 & 3.6 & 5.7 & 4.8 & 4.7 \\
\hline Import volume & -8.5 & 18.6 & 3.3 & -5.5 & 5.1 & 2.6 & 3.9 & 2.5 & 1.9 \\
\hline Terms of trade (deterioration $=-$ ) & 3.3 & -22.2 & 5.6 & 11.6 & 2.7 & -4.5 & -3.6 & 0.2 & -0.9 \\
\hline Real effective exchange rate (depreciation $=-$ ) & 7.0 & -1.8 & 1.4 & -0.5 & 1.2 & 2.1 & 0.4 & 0.4 & 0.2 \\
\hline Nominal exchange rate (CFAF per US\$; average) & 445.7 & 471.0 & 450.0 & 494.4 & 501.7 & 479.2 & 481.6 & 485.3 & 489.4 \\
\hline \multicolumn{10}{|l|}{ Government finances } \\
\hline Domestic revenue (excluding grants) & 30.0 & 2.3 & 21.5 & 26.1 & 10.4 & 11.8 & 9.0 & 8.7 & 7.6 \\
\hline Total expenditure & 26.2 & -6.7 & 16.0 & -0.9 & 6.9 & 14.4 & 10.1 & 8.5 & 6.7 \\
\hline Current expenditure & 7.0 & -4.0 & 13.1 & -2.0 & 7.7 & 12.3 & 6.4 & 9.6 & 6.6 \\
\hline Capital expenditure & 64.7 & -10.1 & 20.2 & 0.7 & 5.8 & 17.4 & 14.9 & 7.1 & 6.7 \\
\hline \multicolumn{10}{|l|}{ Money and credit } \\
\hline Net domestic assets ${ }^{3}$ & 19.7 & -10.9 & 2.0 & 15.9 & 1.6 & 5.4 & 2.1 & 0.4 & 0.5 \\
\hline Credit to government (net) & 8.4 & -10.5 & 0.2 & 7.0 & 0.0 & 0.0 & 0.0 & -1.7 & -1.7 \\
\hline Credit to the economy & 10.1 & 4.3 & 0.8 & 8.0 & 1.6 & 2.1 & 2.1 & 2.1 & 2.2 \\
\hline Velocity (GDP/broad money) & 4.1 & 3.8 & 3.8 & 3.6 & 3.2 & 3.6 & 3.6 & 3.8 & 3.9 \\
\hline & \multicolumn{9}{|c|}{$(\text { Percent of GDP, unless otherwise indicated })^{3}$} \\
\hline Investments and savings & & & & & & & & & \\
\hline Gross investment & 8.7 & 10.1 & 16.3 & 9.8 & 14.9 & 10.9 & 11.2 & 11.2 & 11.2 \\
\hline Of which: government investment & 4.2 & 5.1 & 10.0 & 4.8 & 8.6 & 5.2 & 5.5 & 5.5 & 5.5 \\
\hline Gross domestic savings & -5.3 & -6.6 & 3.7 & -4.0 & 1.1 & -4.0 & -3.8 & -2.8 & -2.2 \\
\hline Of which: government savings & -11.1 & -7.9 & -8.3 & -5.1 & -4.1 & -5.5 & -5.7 & -5.8 & -5.7 \\
\hline Gross national savings & 3.9 & 3.7 & 10.7 & 3.1 & 8.9 & 3.6 & 3.8 & 4.3 & 4.9 \\
\hline \multicolumn{10}{|l|}{ Government finances } \\
\hline Budgetary revenue & 9.2 & 9.0 & 10.3 & 10.8 & 10.7 & 11.1 & 11.4 & 11.6 & 11.7 \\
\hline Total domestic primary expenditure & 12.4 & 11.9 & 14.2 & 12.0 & 12.5 & 13.8 & 14.6 & 14.5 & 14.6 \\
\hline Domestic primary balance & -3.2 & -2.9 & -3.9 & -1.2 & -1.7 & -2.7 & -3.2 & -3.0 & -2.9 \\
\hline \multicolumn{10}{|l|}{ Overall balance (commitment basis) } \\
\hline Including grants & -0.8 & 2.9 & -3.2 & -0.2 & -2.3 & -2.0 & -1.3 & -1.4 & -1.3 \\
\hline Excluding grants & -15.3 & -12.9 & -13.6 & -9.9 & -9.8 & -10.7 & -11.2 & -11.3 & -11.2 \\
\hline External current account (including official current transfers) & -4.9 & -6.4 & -1.3 & -6.7 & -6.0 & -7.3 & -7.4 & -6.9 & -6.3 \\
\hline Excluding official transfers & -11.3 & -14.4 & -5.9 & -10.2 & -8.8 & -11.4 & -11.5 & -11.0 & -10.3 \\
\hline Net present value of external debt/exports of goods and nonfe & & & & & & & & & \\
\hline services (percent) & 364.8 & 419.4 & 111.1 & 93.8 & 134.6 & 91.7 & 99.3 & 99.8 & 94.3 \\
\hline Nominal stock of public debt, including arrears ${ }^{4,5}$ & 167.5 & 157.9 & 54.1 & 50.0 & 62.0 & 47.6 & 46.2 & 43.7 & 40.5 \\
\hline Of which: external debt, including arrears & 132.7 & 121.9 & 28.4 & 20.1 & 34.3 & 20.7 & 21.3 & 21.1 & 19.9 \\
\hline Of which: arrears ${ }^{4}$ & 49.6 & 48.9 & 10.3 & 0.0 & 0.0 & 0.0 & 0.0 & 0.0 & 0.0 \\
\hline & \multicolumn{9}{|c|}{ (US\$ millions, unless otherwise indicated) } \\
\hline \multicolumn{10}{|l|}{ Memorandum items: } \\
\hline Current account balance (including official current transfers) & -41.1 & -53.1 & -11.4 & -56.0 & -51.9 & -68.3 & -73.1 & -72.6 & -69.8 \\
\hline Overall balance of payments & -16.9 & -11.6 & -806.6 & -918.4 & 1.0 & -0.1 & 0.1 & -8.5 & -15.5 \\
\hline Nominal GDP at market prices (CFAF billions) & 377.5 & 393.1 & 418.8 & 413.7 & 443.9 & 447.9 & 476.9 & 510.5 & 544.8 \\
\hline Nominal stock of external arrears, end of period ${ }^{4}$ & 388.6 & 427.5 & 92.8 & 0.0 & 0.0 & 0.0 & 0.0 & 0.0 & 0.0 \\
\hline
\end{tabular}

Sources: Guinea-Bissau authorities; and IMF staff estimates and projections.

${ }^{1}$ As of May 2010 (IMF Country Report No. 10/117).

${ }^{2}$ As of December 2010 (IMF Country Report No. 10/379).

${ }^{3}$ Contribution to the growth of broad money in percent.

${ }^{4}$ Values in 2010 and thereafter reflect assumed impact of HIPC and MDRI debt relief; and estimates for 2010 include Topping Up.

${ }^{5}$ As of end-2010, includes 8.3 percent of GDP in domestic arrears, consisting of pre-1999 arrears (3.3 percent of GDP) and preliminary estimates of the 2000-07 arrears registered at the Treasury (5 percent of GDP). It does not include the preliminary estimates of 2000-07 arrears (13.4 percent of GDP) not registered at the Treasury. 
Table 2. Guinea-Bissau: Monetary Survey, 2008-14

\begin{tabular}{|c|c|c|c|c|c|c|c|c|}
\hline & \multirow[t]{2}{*}{2008} & \multirow[t]{2}{*}{2009} & \multicolumn{2}{|c|}{2010} & \multirow{2}{*}{$\begin{array}{l}2011 \\
\text { Proj. }\end{array}$} & \multirow{2}{*}{$\begin{array}{l}2012 \\
\text { Proj. }\end{array}$} & \multirow{2}{*}{$\begin{array}{l}2013 \\
\text { Proj. }\end{array}$} & \multirow{2}{*}{$\begin{array}{l}2014 \\
\text { Proj }\end{array}$} \\
\hline & & & Prog. $^{1}$ & Proj. & & & & \\
\hline \multicolumn{9}{|c|}{ (CFA billions) } \\
\hline Net foreign assets & 66.6 & 80.6 & 72.6 & 76.6 & 80.1 & 83.7 & 87.1 & 90.5 \\
\hline Central Bank of West African States (BCEAO) & 53.0 & 61.4 & 58.3 & 62.4 & 65.2 & 68.1 & 70.9 & 73.7 \\
\hline Commercial banks & 13.6 & 19.2 & 14.3 & 14.2 & 14.9 & 15.5 & 16.2 & 16.8 \\
\hline Net domestic assets & 33.7 & 22.8 & 36.9 & 39.2 & 45.5 & 48.1 & 48.7 & 49.4 \\
\hline Credit to the government (net) & 19.3 & 8.8 & 9.0 & 16.1 & 16.1 & 16.1 & 13.9 & 11.6 \\
\hline Credit to the private sector & 17.4 & 21.8 & 24.7 & 30.0 & 32.5 & 35.1 & 37.9 & 40.8 \\
\hline Other items (net) & -3.0 & -7.8 & 3.1 & -6.8 & -3.0 & -3.0 & -3.0 & -3.0 \\
\hline Money supply (M2) & 100.3 & 103.4 & 109.5 & 115.9 & 125.6 & 131.8 & 135.7 & 139.9 \\
\hline Currency outside banks & 64.4 & 64.4 & 64.2 & 70.9 & 76.8 & 80.6 & 83.0 & 85.6 \\
\hline Bank deposits & 35.8 & 39.0 & 45.3 & 45.0 & 48.8 & 51.2 & 52.7 & 54.3 \\
\hline Base money (M0) & 64.7 & 66.7 & 76.9 & 74.7 & 81.0 & 85.0 & 87.5 & 90.2 \\
\hline \multicolumn{9}{|l|}{ Contribution to the growth of broad money } \\
\hline Money supply (M2) & 40.0 & 3.1 & 6.0 & 12.0 & 8.4 & 4.9 & 3.0 & 3.1 \\
\hline Net foreign assets & 20.4 & 14.0 & 4.0 & -3.8 & 3.0 & 2.8 & 2.6 & 2.6 \\
\hline BCEAO & 12.0 & 8.3 & 3.2 & 1.0 & 2.4 & 2.3 & 2.1 & 2.1 \\
\hline Commercial banks & 8.4 & 5.6 & 0.8 & -4.8 & 0.6 & 0.5 & 0.5 & 0.5 \\
\hline Net domestic assets & 19.7 & -10.9 & 2.0 & 15.9 & 5.4 & 2.1 & 0.4 & 0.5 \\
\hline Credit to the central government & 8.4 & -10.5 & 0.2 & 7.0 & 0.0 & 0.0 & -1.7 & -1.7 \\
\hline Credit to the private sector & 10.1 & 4.3 & 0.8 & 8.0 & 2.1 & 2.1 & 2.1 & 2.2 \\
\hline Other items net & 1.2 & -4.7 & 1.0 & 0.9 & 3.3 & 0.0 & 0.0 & 0.0 \\
\hline \multicolumn{9}{|l|}{ Memorandum items: } \\
\hline Money supply (M2, / dec t-1) & 40.0 & 3.1 & 6.0 & 12.0 & 8.4 & 4.9 & 3.0 & 3.1 \\
\hline Base money ( $\mathrm{M} 0, /$ dec $\mathrm{t}-1)$ & 21.6 & 25.4 & 6.0 & 12.0 & 8.4 & 4.9 & 3.0 & 3.1 \\
\hline Credit to the private sector $(/ \operatorname{dec} \mathrm{t}-1)$ & 71.5 & 24.9 & 3.6 & 37.9 & 8.3 & 8.0 & 7.9 & 7.8 \\
\hline Velocity (GDP/M2) & 3.8 & 3.8 & 3.8 & 3.6 & 3.6 & 3.6 & 3.8 & 3.9 \\
\hline Money Multiplier (M2/M0) & 1.6 & 1.6 & 1.4 & 1.6 & 1.6 & 1.6 & 1.6 & 1.6 \\
\hline Currency outside banks / M2 (in percent) & 64.3 & 62.3 & 58.7 & 61.2 & 61.2 & 61.2 & 61.2 & 61.2 \\
\hline
\end{tabular}

Sources: BCEAO; and IMF staff estimates and projections.

${ }^{1}$ As of May 2010 (IMF Country Report No. 10/117). 
Table 3. Guinea-Bissau: Balance of Payments, 2008-14

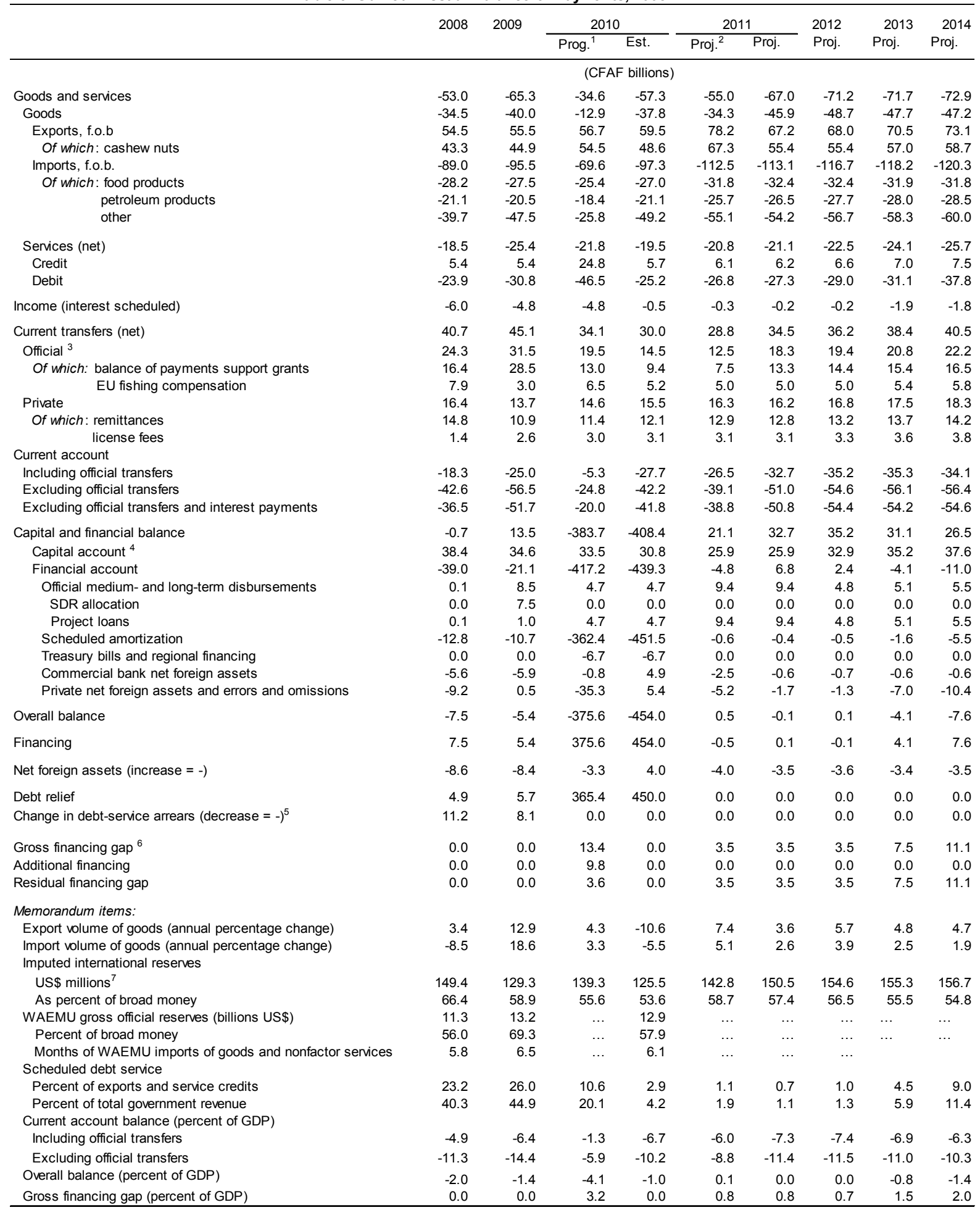

Sources: BCEAO; and IMF staff estimates and projections.

${ }^{1}$ As of May 2010 (IMF Country Report No. 10/117).

${ }^{2}$ As of December 2010 (IMF Country Report No. 10/379).

${ }^{3}$ Includes food aid and technical assistance to projects.

${ }^{4}$ Excludes the financing gap, which BCEAO includes in the capital account.

${ }^{5}$ In 2010, arrears were assumed to be treated at HIPC completion point.

${ }^{6}$ Assumed to be filled with IMF resources and additional donor support.

${ }^{7}$ At program exchange rates. 
Table 4. Guinea-Bissau: Central Government Operations, 2008-14

(CFAF billions)

\begin{tabular}{|c|c|c|c|c|c|c|c|c|c|}
\hline & \multirow[t]{2}{*}{2008} & \multirow[t]{2}{*}{2009} & \multicolumn{2}{|c|}{2010} & \multicolumn{2}{|c|}{2011} & \multirow{2}{*}{$\begin{array}{r}2012 \\
\text { Proj. }\end{array}$} & \multirow{2}{*}{$\begin{array}{c}2013 \\
\text { Proj. }\end{array}$} & \multirow{2}{*}{$\begin{array}{r}2014 \\
\text { Proj. }\end{array}$} \\
\hline & & & Prog. $^{1}$ & Est. & Prog. $^{2}$ & Proj. & & & \\
\hline Revenue and grants & 89.3 & 97.5 & 86.6 & 84.6 & 80.9 & 88.8 & 101.4 & 109.5 & 117.3 \\
\hline Revenue & 34.6 & 35.4 & 43.0 & 44.6 & 47.7 & 49.9 & 54.4 & 59.1 & 63.6 \\
\hline Tax revenue & 20.9 & 26.6 & 30.1 & 33.1 & 35.7 & 37.9 & 42.0 & 45.8 & 49.4 \\
\hline Nontax revenue & 13.7 & 8.8 & 12.9 & 11.5 & 11.9 & 11.9 & 12.4 & 13.3 & 14.2 \\
\hline Fishing compensation ${ }^{3}$ & 7.9 & 3.0 & 6.5 & 5.2 & 5.0 & 5.0 & 5.0 & 5.4 & 5.8 \\
\hline Grants & 54.7 & 62.1 & 43.6 & 40.0 & 33.2 & 38.9 & 47.1 & 50.4 & 53.8 \\
\hline Budget support ${ }^{4}$ & 16.4 & 28.5 & 13.0 & 9.4 & 7.5 & 13.3 & 14.4 & 15.4 & 16.5 \\
\hline Project grants & 38.4 & 33.6 & 30.6 & 30.6 & 25.7 & 25.7 & 32.7 & 35.0 & 37.3 \\
\hline Total expenditure & 92.4 & 86.3 & 100.1 & 85.5 & 91.3 & 97.9 & 107.7 & 116.9 & 124.6 \\
\hline Current expenditure & 52.4 & 50.3 & 56.9 & 49.3 & 52.9 & 55.3 & 58.9 & 64.5 & 68.8 \\
\hline Wages and salaries & 20.5 & 20.5 & 20.7 & 20.7 & 22.4 & 23.8 & 25.3 & 26.9 & 28.7 \\
\hline Goods and services & 8.0 & 6.4 & 10.2 & 8.6 & 8.1 & 9.1 & 9.7 & 10.4 & 11.1 \\
\hline Transfers & 10.9 & 11.0 & 12.7 & 10.7 & 12.4 & 12.6 & 13.4 & 14.3 & 15.6 \\
\hline Other current expenditures ${ }^{5}$ & 5.7 & 7.5 & 7.8 & 8.6 & 9.0 & 9.0 & 9.6 & 10.3 & 11.0 \\
\hline Scheduled interest & 7.3 & 4.9 & 5.4 & 0.7 & 0.9 & 0.8 & 0.9 & 2.6 & 2.4 \\
\hline Domestic interest & 1.3 & 0.0 & 0.6 & 0.2 & 0.6 & 0.6 & 0.6 & 0.7 & 0.7 \\
\hline External interest & 6.0 & 4.8 & 4.8 & 0.5 & 0.3 & 0.2 & 0.2 & 1.9 & 1.8 \\
\hline Capital expenditure and net lending & 40.0 & 36.0 & 43.3 & 36.2 & 38.4 & 42.5 & 48.9 & 52.3 & 55.9 \\
\hline Public investment program & 39.6 & 35.7 & 42.0 & 35.8 & 37.8 & 41.9 & 47.2 & 50.6 & 54.0 \\
\hline Domestically financed & 1.2 & 1.0 & 6.6 & 0.5 & 2.7 & 6.8 & 9.8 & 10.5 & 11.2 \\
\hline Foreign financed & 38.4 & 34.6 & 35.4 & 35.4 & 35.1 & 35.1 & 37.5 & 40.1 & 42.8 \\
\hline Other capital expenditure & 0.4 & 0.3 & 1.2 & 0.4 & 0.6 & 0.6 & 1.6 & 1.8 & 1.9 \\
\hline Domestically financed & 0.4 & 0.3 & 1.2 & 0.4 & 0.6 & 0.6 & 1.6 & 1.8 & 1.9 \\
\hline Overall balance, including grants (commitment) & -3.1 & 11.2 & -13.5 & -0.9 & -10.4 & -9.1 & -6.3 & -7.4 & -7.3 \\
\hline Overall balance, excluding grants (commitment) & -57.8 & -50.9 & -57.1 & -40.9 & -43.6 & -48.0 & -53.4 & -57.8 & -61.1 \\
\hline Net domestic arrears & 4.8 & -4.9 & -7.9 & -7.7 & -1.9 & -3.5 & -1.5 & -1.5 & -1.5 \\
\hline Accumulation current year & 10.7 & 0.0 & 0.0 & 0.0 & 0.0 & 0.0 & 0.0 & 0.0 & 0.0 \\
\hline Payment previous years & -5.9 & -4.9 & -7.9 & -7.7 & -1.9 & -3.5 & -1.5 & -1.5 & -1.5 \\
\hline External interest arrears current year & 5.2 & 4.3 & 0.0 & 0.0 & 0.0 & 0.0 & 0.0 & 0.0 & 0.0 \\
\hline Float and statistical discrepancies & -0.4 & 0.0 & 0.0 & -1.8 & 0.0 & 0.0 & 0.0 & 0.0 & 0.0 \\
\hline Overall balance, including grants (cash) & 6.4 & 10.7 & -21.5 & -10.5 & -12.3 & -12.6 & -7.8 & -8.9 & -8.8 \\
\hline Financing & -6.4 & -10.7 & 21.5 & 10.5 & 12.3 & 12.6 & 7.8 & 8.9 & 8.8 \\
\hline Domestic financing & -4.6 & -10.5 & 0.2 & 7.2 & 0.0 & 0.0 & 0.0 & -2.2 & -2.3 \\
\hline Bank financing & -4.6 & -10.5 & 0.2 & 7.2 & 0.0 & 0.0 & 0.0 & -2.2 & -2.3 \\
\hline Of which: Domestic banks & -5.3 & -2.5 & -4.2 & -3.7 & 0.0 & 0.0 & 0.0 & 0.0 & 0.0 \\
\hline Regional (including T-bills) & 0.0 & 0.0 & -6.7 & -6.7 & 0.0 & 0.0 & 0.0 & 0.0 & 0.0 \\
\hline $\mathrm{BCEAO}^{6}$ & 0.7 & -8.0 & 11.2 & 17.7 & 0.0 & 0.0 & 0.0 & -2.2 & -2.3 \\
\hline Foreign financing (net) & -1.8 & -0.2 & 7.8 & 3.2 & 8.8 & 9.1 & 4.3 & 3.5 & 0.0 \\
\hline Disbursements & 0.1 & 1.0 & 4.7 & 4.7 & 9.4 & 9.4 & 4.8 & 5.1 & 5.5 \\
\hline Projects & 0.1 & 1.0 & 4.7 & 4.7 & 9.4 & 9.4 & 4.8 & 5.1 & 5.5 \\
\hline Amortization (scheduled and arrears) & -12.8 & -10.7 & -362.4 & -451.5 & -0.6 & -0.4 & -0.5 & -1.6 & -5.5 \\
\hline External arrears ${ }^{7}$ & 6.0 & 3.7 & 0.0 & 0.0 & 0.0 & 0.0 & 0.0 & 0.0 & 0.0 \\
\hline Debt relief & 4.9 & 5.7 & 365.4 & 450.0 & 0.0 & 0.0 & 0.0 & 0.0 & 0.0 \\
\hline Gross financing gap $(+=$ financing need $)$ & 0.0 & 0.0 & 13.4 & 0.0 & 3.5 & 3.5 & 3.5 & 7.6 & 11.1 \\
\hline Additional financing ${ }^{8}$ & 0.0 & 0.0 & 9.8 & 0.0 & 0.0 & 0.0 & 0.0 & 0.0 & 0.0 \\
\hline Residual financing gap ${ }^{9}$ & 0.0 & 0.0 & 3.6 & 0.0 & 3.5 & 3.5 & 3.5 & 7.6 & 11.1 \\
\hline Domestic primary balance & -12.1 & -11.4 & -16.3 & -4.9 & -7.6 & -12.1 & -15.1 & -15.1 & -15.8 \\
\hline Revenue & 34.6 & 35.4 & 43.0 & 44.6 & 47.7 & 49.9 & 54.4 & 59.1 & 63.6 \\
\hline Primary expenditure & 46.7 & 46.8 & 59.3 & 49.5 & 55.3 & 61.9 & 69.4 & 74.2 & 79.4 \\
\hline
\end{tabular}

Sources: Guinea-Bissau authorities; and IMF staff estimates and projections.

${ }^{1}$ As of May 2010 (IMF Country Report No. 10/117).

${ }^{2}$ As of December 2010 (IMF Country Report No. 10/379).

${ }^{3}$ In 2010, includes the disbursement of one tranche of EU fishing compensation, compared to two tranches assumed under the program.

${ }^{4}$ In 2009 includes CFAF 5.2 billion ( $€ 8$ million) of V-Flex 2009 from the EU for payment of 2008 arrears.

${ }^{5}$ Beginning in 2012, incorporates severance payments for excess employees identified under the action plan for public administration reform.

${ }^{6}$ Beginning in 2013, assumes payment of scheduled domestic debt owed to the BCEAO and rescheduling of scheduled interest and amortization falling due in 2010 and principal falling due in 2011 and 2012 with terms similar to previous reschedullings.

${ }^{7}$ In 2010, arrears are assumed to be treated as debt relief at HIPC completion point.

${ }^{8}$ In 2010 covered with the EU V-Flex.

${ }^{9}$ Assumed to be covered with IMF resources under the ECF arrangement until 2012. 
Table 4. Guinea-Bissau: Central Government Operations, 2008-14 (concluded)

\begin{tabular}{|c|c|c|c|c|c|c|c|c|c|}
\hline & \multirow[t]{2}{*}{2008} & \multirow[t]{2}{*}{2009} & \multicolumn{2}{|c|}{2010} & \multicolumn{2}{|c|}{2011} & \multirow{2}{*}{$\begin{array}{r}2012 \\
\text { Proj. }\end{array}$} & \multirow{2}{*}{$\begin{array}{r}2013 \\
\text { Proj. }\end{array}$} & \multirow{2}{*}{$\begin{array}{r}2014 \\
\text { Proj. }\end{array}$} \\
\hline & & & Prog. ${ }^{1}$ & Est. & Prog. $^{2}$ & Proj. & & & \\
\hline Revenue and grants & 23.7 & 24.8 & 20.7 & 20.4 & 18.2 & 19.8 & 21.3 & 21.4 & 21.5 \\
\hline Revenue & 9.2 & 9.0 & 10.3 & 10.8 & 10.7 & 11.1 & 11.4 & 11.6 & 11.7 \\
\hline Tax revenue & 5.5 & 6.8 & 7.2 & 8.0 & 8.1 & 8.5 & 8.8 & 9.0 & 9.1 \\
\hline Grants & 14.5 & 15.8 & 10.4 & 9.7 & 7.5 & 8.7 & 9.9 & 9.9 & 9.9 \\
\hline Budget support ${ }^{4}$ & 4.3 & 7.3 & 3.1 & 2.3 & 1.7 & 3.0 & 3.0 & 3.0 & 3.0 \\
\hline Project grants & 10.2 & 8.5 & 7.3 & 7.4 & 5.8 & 5.7 & 6.8 & 6.8 & 6.8 \\
\hline Total expenditure & 24.5 & 21.9 & 23.9 & 20.7 & 20.6 & 21.8 & 22.6 & 22.9 & 22.9 \\
\hline Current expenditure & 13.9 & 12.8 & 13.6 & 11.9 & 11.9 & 12.4 & 12.3 & 12.6 & 12.6 \\
\hline Scheduled interest & 1.9 & 1.2 & 1.3 & 0.2 & 0.2 & 0.2 & 0.2 & 0.5 & 0.4 \\
\hline Domestic interest & 0.3 & 0.0 & 0.2 & 0.1 & 0.1 & 0.1 & 0.1 & 0.1 & 0.1 \\
\hline External interest & 1.6 & 1.2 & 1.1 & 0.1 & 0.1 & 0.0 & 0.0 & 0.4 & 0.3 \\
\hline Capital expenditure and net lending & 10.6 & 9.2 & 10.3 & 8.8 & 8.6 & 9.5 & 10.3 & 10.3 & 10.3 \\
\hline Public investment program & 10.5 & 9.1 & 10.0 & 8.7 & 8.5 & 9.4 & 9.9 & 9.9 & 9.9 \\
\hline Domestically financed & 0.3 & 0.3 & 1.6 & 0.1 & 0.6 & 1.5 & 2.1 & 2.1 & 2.1 \\
\hline Foreign financed & 10.2 & 8.8 & 8.4 & 8.5 & 7.9 & 7.8 & 7.9 & 7.9 & 7.9 \\
\hline Other capital expenditure & 0.1 & 0.1 & 0.3 & 0.1 & 0.1 & 0.1 & 0.3 & 0.3 & 0.3 \\
\hline Domestically financed & 0.1 & 0.1 & 0.3 & 0.1 & 0.1 & 0.1 & 0.3 & 0.3 & 0.3 \\
\hline Overall balance, including grants (commitment) & -0.8 & 2.9 & -3.2 & -0.2 & -2.3 & -2.0 & -1.3 & -1.4 & -1.3 \\
\hline Overall balance, excluding grants (commitment) & -15.3 & -12.9 & -13.6 & -9.9 & -9.8 & -10.7 & -11.2 & -11.3 & -11.2 \\
\hline Financing & -1.7 & -2.7 & 5.1 & 2.5 & 2.8 & 2.8 & 1.6 & 1.7 & 1.6 \\
\hline Domestic financing & -1.2 & -2.7 & 0.1 & 1.8 & 0.0 & 0.0 & 0.0 & -0.4 & -0.4 \\
\hline Bank financing & -1.2 & -2.7 & 0.1 & 1.8 & 0.0 & 0.0 & 0.0 & -0.4 & -0.4 \\
\hline Of which: Domestic banks & -1.4 & -0.6 & -1.0 & -0.9 & 0.0 & 0.0 & 0.0 & 0.0 & 0.0 \\
\hline Regional (including T-bills) & 0.0 & 0.0 & -1.6 & -1.6 & 0.0 & 0.0 & 0.0 & 0.0 & 0.0 \\
\hline $\mathrm{BCEAO}^{6}$ & 0.2 & -2.0 & 2.7 & 4.3 & 0.0 & 0.0 & 0.0 & -0.4 & -0.4 \\
\hline Foreign financing (net) & -0.5 & -0.1 & 1.9 & 0.8 & 2.0 & 2.0 & 0.9 & 0.7 & 0.0 \\
\hline Disbursements & 0.0 & 0.3 & 1.1 & 1.1 & 2.1 & 2.1 & 1.0 & 1.0 & 1.0 \\
\hline Projects & 0.0 & 0.3 & 1.1 & 1.1 & 2.1 & 2.1 & 1.0 & 1.0 & 1.0 \\
\hline Amortization (scheduled and arrears) & -3.4 & -2.7 & -86.5 & -109.1 & -0.1 & -0.1 & -0.1 & -0.3 & -1.0 \\
\hline External arrears ${ }^{7}$ & 1.6 & 1.0 & 0.0 & 0.0 & 0.0 & 0.0 & 0.0 & 0.0 & 0.0 \\
\hline Debt relief & 1.3 & 1.4 & 87.3 & 108.8 & 0.0 & 0.0 & 0.0 & 0.0 & 0.0 \\
\hline Gross financing gap $(+=$ financing need $)$ & 0.0 & 0.0 & 3.2 & 0.0 & 0.8 & 0.8 & 0.7 & 1.5 & 2.0 \\
\hline Additional financing ${ }^{8}$ & 0.0 & 0.0 & 2.3 & 0.0 & 0.0 & 0.0 & 0.0 & 0.0 & 0.0 \\
\hline Residual financing gap ${ }^{9}$ & 0.0 & 0.0 & 0.9 & 0.0 & 0.8 & 0.8 & 0.7 & 1.5 & 2.0 \\
\hline Domestic primary balance & -3.2 & -2.9 & -3.9 & -1.2 & -1.7 & -2.7 & -3.2 & -3.0 & -2.9 \\
\hline Revenue & 9.2 & 9.0 & 10.3 & 10.8 & 10.7 & 11.1 & 11.4 & 11.6 & 11.7 \\
\hline Primary expenditure & 12.4 & 11.9 & 14.2 & 12.0 & 12.5 & 13.8 & 14.6 & 14.5 & 14.6 \\
\hline
\end{tabular}

Sources: Guinea-Bissau authorities; and IMF staff estimates and projections.

${ }^{1}$ As of May 2010 (IMF Country Report No. 10/117).

2 As of December 2010 (IMF Country Report No. 10/379).

${ }^{3}$ In 2010, includes the disbursement of one tranche of EU fishing compensation, compared to two tranches assumed under the program.

${ }^{4}$ In 2009 includes CFAF 5.2 billion ( $€ 8$ million) of V-Flex 2009 from the EU for payment of 2008 arrears.

${ }^{5}$ Beginning in 2012, incorporates severance payments for excess employees identified under the action plan for public administration reform.

${ }^{6}$ Beginning in 2013, assumes payment of scheduled domestic debt owed to the BCEAO and rescheduling of scheduled interest and amortization falling due in 2010 and principal falling due in 2011 and 2012 with terms similar to previous reschedullings.

${ }^{7}$ In 2010, arrears are assumed to be treated as debt relief at HIPC completion point.

${ }^{8}$ In 2010 covered with the EU V-Flex.

${ }^{9}$ Assumed to be covered with IMF resources under the ECF arrangement until 2012. 
Table 5. Guinea-Bissau: Quantitative Indicators for the ECF Program for 2010

Quarterly Targets ${ }^{1}$

(Cumulative, CFAF millions)

\begin{tabular}{|c|c|c|c|c|c|c|c|c|}
\hline & \multicolumn{2}{|c|}{ End-March } & \multicolumn{2}{|c|}{ End-June } & \multicolumn{2}{|c|}{ End-Sept. } & \multicolumn{2}{|c|}{ End-Dec. } \\
\hline & Prog. $^{2}$ & $\overline{\text { Actual }}$ & Prog. $^{2}$ & Actual & Prog. $^{2}$ & Actual & Prog. $^{2}$ & Prel. \\
\hline \multicolumn{9}{|l|}{ Performance criteria ${ }^{3}$} \\
\hline 1. Domestic financing of the budget & 10,618 & 5,975 & 8,627 & 302 & 8,736 & 4,308 & 12,231 & 3,409 \\
\hline 2. New domestic arrears & 0 & 0 & 0 & 0 & 0 & 0 & 0 & 0 \\
\hline 3. External nonconcessional public borrowing, maturity $>1$ year & 0 & 0 & 0 & 0 & 0 & 0 & 0 & 0 \\
\hline 4. External short term public borrowing & 0 & 0 & 0 & 0 & 0 & 0 & 0 & 0 \\
\hline 5. New external payment arrears & 0 & 0 & 0 & 0 & 0 & 0 & 0 & 0 \\
\hline \multicolumn{9}{|l|}{ Indicative targets } \\
\hline 6. Government tax revenue & 5,420 & 6,391 & 15,148 & 17,538 & 24,218 & 25,394 & 30,101 & 33,086 \\
\hline 7. Domestic primary balance (commitment basis) & $-7,789$ & $-2,401$ & $-8,386$ & 1,048 & $-11,030$ & $-1,352$ & $-17,938$ & $-4,865$ \\
\hline 8. Nonregularized expenditure (DNTs) & 200 & 0 & 200 & 0 & 200 & 0 & 200 & 0 \\
\hline 9. Social and priority spending & 4,062 & 1,989 & 8,123 & 4,223 & 12,185 & 6,818 & $8,647^{4}$ & 9,152 \\
\hline
\end{tabular}

${ }^{1}$ Cumulative from January 1. The definition of the aggregates and adjustors is provided in the Technical Memorandum of Understanding (TMU).

${ }^{2}$ Adjusted target under the program.

${ }^{3}$ Performance criteria (PC) are defined for June and December. March and September are indicative. All performance criteria (PC) are ceillings and PC 3,4 , and 5 apply continously.

${ }^{4}$ Indicative target for social and priority spending was revised compared to program (IMF Country Report No. 10/379) from CFAF 16,246 to CFAF 8,647. 
Table 6. Guinea-Bissau: Structural Benchmarks Under the ECF Second Review

\begin{tabular}{|c|c|c|c|c|c|c|}
\hline Category & & Structural Benchmarks & $\begin{array}{c}\text { Macro } \\
\text { Rationale }\end{array}$ & $\begin{array}{l}\text { Actual } \\
\text { Delivery } \\
\text { Date }\end{array}$ & Ministry & $\begin{array}{l}\text { Status of } \\
\text { Implementa- } \\
\text { tion }\end{array}$ \\
\hline $\begin{array}{l}\text { Fiscal } \\
\text { management }\end{array}$ & 1 & $\begin{array}{l}\text { Establish an operational link from } \\
\text { budget/treasury under the SIGFIP to } \\
\text { the customs and tax administration. }\end{array}$ & $\begin{array}{l}\text { Greater control } \\
\text { in budget } \\
\text { execution }\end{array}$ & March 2011 & $\mathrm{MoF}^{1}$ & Met \\
\hline $\begin{array}{l}\text { Expenditure } \\
\text { reforms }\end{array}$ & 2 & $\begin{array}{l}\text { Submit to the Council of Ministers an } \\
\text { action plan for public administration } \\
\text { reform, with a medium-term schedule } \\
\text { to downsize the civil service through } \\
\text { retirement and removing redundant } \\
\text { workers beginning in } 2011 \text {. }\end{array}$ & $\begin{array}{l}\text { To improve the } \\
\text { efficiency of civil } \\
\text { service }\end{array}$ & Jan 2011 & $\begin{array}{l}\text { MoF } \\
\text { MinLaPS }^{2}\end{array}$ & Met \\
\hline
\end{tabular}


Table 7. Guinea-Bissau: External Debt Indicators ${ }^{1}$

\begin{tabular}{lcccc}
\hline & Threshold $^{2}$ & $\mathbf{2 0 1 1}$ & $\mathbf{2 0 2 0}$ & $\mathbf{2 0 3 0}$ \\
\hline PV of debt to GDP & 30 & 15.0 & 11.1 & 10.7 \\
PV of debt to exports & 100 & 91.7 & 78.6 & 78.1 \\
PV of debt to revenue & 200 & 135.0 & 92.7 & 86.4 \\
Debt service to exports & 15 & 0.7 & 5.2 & 5.4 \\
Debt service to revenue & 25 & 1.1 & 6.1 & 6.0 \\
\hline
\end{tabular}

Source: IMF staff estimates.

${ }^{1}$ Debt indicators refer to Guinea-Bissau's public and publicly guaranteed external debt, assuming full delivery of HIPC, beyond-HIPC, topping up, and MDRI assistance.

${ }^{2}$ Threshold over which countries with weak policy and institutional frameworks would have at least a 25 percent chance of having a prolonged debt distress episode in the coming year. With an average Country Policy and Institutional Assessment (CPIA) rating of 2.58 over 2007-09, Guinea-Bissau is classified as having a weak policy and institutional framework. 
Table 8. Guinea-Bissau: Quantitative Indicators for the ECF Program for 2011

Quarterly Targets ${ }^{1}$

(Cumulative, CFAF millions)

\begin{tabular}{|c|c|c|c|}
\hline End-March & End-June & End-Sept. & End-Dec. \\
\hline Prog. & Prog. & Prog. & Prog. \\
\hline
\end{tabular}

\section{Performance criteria ${ }^{2}$}

1. Domestic financing of the budget

2. New domestic arrears

3. External nonconcessional public borrowing, maturity $>1$ year

4. External short term public borrowing

5. New external payment arrears

Indicative targets ${ }^{3}$

$\begin{array}{lrrrr}\text { 6. Government tax revenue } & 6,437 & 17,988 & 30,506 & 37,917 \\ \text { 7. Domestic primary balance (commitment basis) } & -3,597 & -5,842 & -5,911 & -12,087 \\ \text { 8. Nonregularized expenditure (DNTs) } & 200 & 200 & 200 & 200 \\ \text { 9. Social and priority spending } & 2,932 & 5,864 & 8,795 & 12,227\end{array}$

${ }^{1}$ Cumulative from January 1 . The definition of the aggregates and adjustors is provided in the Technical Memorandum of Understanding (TMU).

${ }^{2}$ Performance criteria (PC) are defined for June and December. March and September are indicative. All PC are ceillings and PC 3, 4, and 5 apply continously.

${ }^{3}$ The indicative targets 6, 7 and 9 were revised compared to program (IMF Country Report No. 10/379) to reflect the higher revenue base from 2010 and the additional budget support. 
Table 9. Guinea-Bissau: Structural Benchmarks Under the ECF Third and Fourth Review

\begin{tabular}{|c|c|c|c|c|c|}
\hline Category & & Structural Benchmarks & Macro Rationale & $\begin{array}{l}\text { Delivery } \\
\text { Date }\end{array}$ & Ministry \\
\hline \multicolumn{6}{|c|}{ Third Review } \\
\hline \multirow{3}{*}{$\begin{array}{l}\text { Tax reform } \\
\text { and } \\
\text { strengthening } \\
\text { revenue } \\
\text { collection }\end{array}$} & 1 & $\begin{array}{l}\text { Customize and test ASYCUDA ++, } \\
\text { and provide training to staff. }\end{array}$ & $\begin{array}{l}\text { To strengthen } \\
\text { revenue } \\
\text { collection }\end{array}$ & August 2011 & $\mathrm{MoF}^{1}$ \\
\hline & 2 & $\begin{array}{l}\text { Conduct a comprehensive review } \\
\text { of all customs exemptions to } \\
\text { further broaden the customs } \\
\text { revenue base and submit report to } \\
\text { Council of Ministers. }\end{array}$ & $\begin{array}{l}\text { To strengthen } \\
\text { revenue } \\
\text { collection }\end{array}$ & June 2011 & MoF \\
\hline & 3 & $\begin{array}{l}\text { Cross-check ASYCUDA data with } \\
\text { tax returns for } 300 \text { largest } \\
\text { importers in 2009-10. }\end{array}$ & $\begin{array}{l}\text { To strengthen } \\
\text { revenue } \\
\text { collection }\end{array}$ & June 2011 & MoF \\
\hline $\begin{array}{l}\text { Expenditure } \\
\text { reforms }\end{array}$ & 4 & $\begin{array}{l}\text { Extend the computerized and } \\
\text { unified payroll and personnel } \\
\text { management system to all } \\
\text { ministries. }\end{array}$ & $\begin{array}{l}\text { To modernize } \\
\text { public } \\
\text { administration }\end{array}$ & August 2011 & $\begin{array}{l}\text { MoF } \\
\text { MinLaPS }\end{array}$ \\
\hline $\begin{array}{l}\text { Business } \\
\text { environment }\end{array}$ & 5 & $\begin{array}{l}\text { Prepare an action plan to identify } \\
\text { and remove further impediments to } \\
\text { private sector development and } \\
\text { improve the ease of doing } \\
\text { business in Guinea-Bissau. }\end{array}$ & $\begin{array}{l}\text { Improve the ease } \\
\text { of doing business }\end{array}$ & August 2011 & $\operatorname{MinE} \operatorname{con}^{3}$ \\
\hline $\begin{array}{l}\text { Public debt } \\
\text { management }\end{array}$ & 6 & $\begin{array}{l}\text { Start producing, on a quarterly } \\
\text { basis, electronic reports on public } \\
\text { debt (external and domestic). }\end{array}$ & $\begin{array}{l}\text { Improve } \\
\text { transparency }\end{array}$ & June 2011 & MoF \\
\hline \multicolumn{6}{|c|}{ Fourth Review } \\
\hline $\begin{array}{l}\text { Public debt } \\
\text { management }\end{array}$ & 1 & $\begin{array}{l}\text { Use SYGADE to record, monitor, } \\
\text { and manage all public debt } \\
\text { (external and domestic) }\end{array}$ & $\begin{array}{l}\text { Improve debt } \\
\text { management }\end{array}$ & Dec 2011 & MoF \\
\hline \multirow{2}{*}{$\begin{array}{l}\text { Tax reform } \\
\text { and } \\
\text { strengthening } \\
\text { revenue } \\
\text { collection }\end{array}$} & 2 & $\begin{array}{l}\text { Audit those importers } \\
\text { underreporting their tax liabilities, } \\
\text { beginning with largest gaps. }\end{array}$ & $\begin{array}{l}\text { To strengthen } \\
\text { revenue } \\
\text { collection }\end{array}$ & Dec 2011 & MoF \\
\hline & 3 & $\begin{array}{l}\text { Review the legal framework that } \\
\text { regulates revenue sharing } \\
\text { agreements ("restitutions"). }\end{array}$ & $\begin{array}{l}\text { To strengthen } \\
\text { revenue } \\
\text { collection }\end{array}$ & Dec 2011 & MoF \\
\hline $\begin{array}{l}\text { Business } \\
\text { environment }\end{array}$ & 4 & $\begin{array}{l}\text { Prepare a plan to manage the } \\
\text { country's natural resources. }\end{array}$ & $\begin{array}{l}\text { Improve the ease } \\
\text { of doing business }\end{array}$ & Dec 2011 & MinEcon \\
\hline
\end{tabular}

\footnotetext{
${ }^{1}$ Ministry of Finance

${ }^{2}$ Ministry of Labor and Public Administration

${ }^{3}$ Ministry of Economy
} 
Table 10. Guinea-Bissau: Reviews and Disbursements under the Three-Year ECF Arrangement, 2010-13

\begin{tabular}{lllcc}
\hline \multicolumn{1}{c}{ Board Date } & \multicolumn{1}{c}{ Test Date } & \multicolumn{1}{c}{ Action } & \multicolumn{2}{c}{ Disbursement } \\
\hline & & & \% of quota & SDR millions \\
May 7, 2010 & & Board approval & $55.5^{1}$ & $7.881^{1}$ \\
December,13 2010 & End-June 2010 & First review & 17 & 2.414 \\
May 2011 & End-December 2010 & Second review & 17 & 2.414 \\
December 2011 & End-June 2011 & Third review & 17 & 2.414 \\
May 2012 & End-December 2011 & Fourth review & 17 & 2.414 \\
December 2012 & End-June 2012 & Fifth review & 17 & 2.414 \\
May 2013 & End-December 2012 & Sixth review & 17 & 2.414 \\
\hline Total & & & 157.5 & 22.365 \\
Source: IMF estimates. & & & & \\
11 For the amount approved for disbursement at the time of Board approval of the arrangement, 37.5 percent of \\
quota (SDR 5.325 million), was approved to immediately repurchase previously provided EPCA credit.
\end{tabular}


Table 11. Guinea-Bissau: Indicators of Capacity to Repay the Fund, 2008-18

\begin{tabular}{|c|c|c|c|c|c|c|c|c|c|c|c|}
\hline & 2008 & 2009 & 2010 & 2011 & 2012 & 2013 & 2014 & 2015 & 2016 & 2017 & 2018 \\
\hline & & & & \multicolumn{8}{|c|}{ Projections } \\
\hline \multicolumn{12}{|l|}{$\begin{array}{l}\text { Fund obligations based on existing credit }{ }^{1} \\
\text { (SDR millions) }\end{array}$} \\
\hline Principal & 1.3 & 1.0 & 14.2 & 0.0 & 0.0 & 0.0 & 0.0 & 0.0 & 0.5 & 0.5 & 0.5 \\
\hline Charges and interest ${ }^{2}$ & 0.1 & 0.1 & 0.0 & 0.0 & 0.0 & 0.0 & 0.0 & 0.0 & 0.0 & 0.0 & 0.0 \\
\hline \multicolumn{12}{|l|}{$\begin{array}{l}\text { Fund obligations based on existing and prospective } \text { credit }^{3} \\
\text { (SDR millions) }\end{array}$} \\
\hline Principal & 1.3 & 1.0 & 14.2 & 0.0 & 0.0 & 0.0 & 0.0 & 0.0 & 0.7 & 1.7 & 2.7 \\
\hline Charges and interest & 0.1 & 0.1 & 0.0 & 0.0 & 0.0 & 0.0 & 0.0 & 0.0 & 0.0 & 0.0 & 0.0 \\
\hline \multicolumn{12}{|l|}{ Total obligations based on existing and prospective credit ${ }^{3,5}$} \\
\hline SDR millions & 1.4 & 1.1 & 14.3 & 0.0 & 0.0 & 0.0 & 0.0 & 0.0 & 0.8 & 1.7 & 2.7 \\
\hline CFAF billions & 1.0 & 0.8 & 10.8 & 0.0 & 0.0 & 0.0 & 0.0 & 0.0 & 0.4 & 0.8 & 1.3 \\
\hline Percent government revenue & 2.8 & 2.2 & 24.1 & 0.0 & 0.0 & 0.0 & 0.0 & 0.0 & 0.5 & 1.1 & 1.6 \\
\hline Percent exports of goods and services & 1.8 & 1.4 & 18.1 & 0.0 & 0.0 & 0.0 & 0.0 & 0.0 & 0.5 & 1.0 & 1.5 \\
\hline Percent debt service $^{4}$ & 4.9 & 5.0 & 2.4 & 0.2 & 2.7 & 0.8 & 0.2 & 0.2 & 5.0 & 18.8 & 27.4 \\
\hline Percent GDP & 0.3 & 0.2 & 2.6 & 0.0 & 0.0 & 0.0 & 0.0 & 0.0 & 0.1 & 0.1 & 0.2 \\
\hline Percent quota & 9.7 & 7.7 & 100.4 & 0.0 & 0.2 & 0.3 & 0.3 & 0.3 & 5.3 & 12.2 & 18.8 \\
\hline Percent reserves & 1.5 & 1.3 & 17.3 & 0.0 & 0.0 & 0.0 & 0.0 & 0.0 & 0.5 & 1.0 & 1.4 \\
\hline \multicolumn{12}{|l|}{ Outstanding Fund credit ${ }^{3,5}$} \\
\hline SDR millions & 5.6 & 6.3 & 2.4 & 7.2 & 12.1 & 14.5 & 14.5 & 14.5 & 13.8 & 12.1 & 9.4 \\
\hline CFAF billions & 3.9 & 4.6 & 1.8 & 5.4 & 9.1 & 11.0 & 7.1 & 7.1 & 6.7 & 5.9 & 4.6 \\
\hline Percent government revenue & 11.4 & 13.0 & 4.1 & 10.9 & 16.7 & 18.6 & 11.1 & 10.4 & 9.2 & 7.6 & 5.5 \\
\hline Percent exports of goods and services & 7.2 & 8.3 & 3.1 & 8.1 & 13.4 & 15.6 & 9.7 & 9.3 & 8.4 & 7.0 & 5.1 \\
\hline Percent debt service $^{4}$ & 19.9 & 29.0 & 0.4 & 1026.9 & 1248.0 & 315.0 & 97.5 & 99.9 & 90.7 & 131.1 & 96.4 \\
\hline Percent GDP & 1.0 & 1.2 & 0.4 & 1.2 & 1.9 & 2.1 & 1.3 & 1.2 & 1.1 & 0.9 & 0.7 \\
\hline Percent quota & 39.3 & 44.7 & 17.0 & 51.0 & 85.0 & 102.0 & 102.0 & 102.0 & 96.9 & 85.0 & 66.3 \\
\hline Percent reserves & 5.9 & 7.6 & 2.9 & 7.5 & 12.2 & 14.6 & 9.2 & 9.0 & 8.4 & 6.9 & 5.1 \\
\hline Net use of Fund credit (SDR millions) & 2.3 & 0.8 & 5.0 & 4.8 & 4.8 & 2.4 & 0.0 & 0.0 & -0.7 & -1.7 & -2.7 \\
\hline Disbursements & 3.6 & 1.8 & 10.3 & 4.8 & 4.8 & 2.4 & 0.0 & 0.0 & 0.0 & 0.0 & 0.0 \\
\hline HIPC and MDRI debt relief ${ }^{5}$ & 0.0 & 0.0 & 8.9 & & & & & & & & \\
\hline Repayments and repurchases before HIPC and MDRI & 1.3 & 1.0 & 14.2 & 0.0 & 0.0 & 0.0 & 0.0 & 0.0 & 0.7 & 1.7 & 2.7 \\
\hline Repayments and repurchases after HIPC and MDRI & 1.3 & 1.0 & 5.3 & 0.0 & 0.0 & 0.0 & 0.0 & 0.0 & 0.7 & 1.7 & 2.7 \\
\hline & & & & & (CFAF & billions & & & & & \\
\hline \multicolumn{12}{|l|}{ Memorandum items: } \\
\hline Charges and interest, after assumed subsidies & 0.1 & 0.0 & 0.0 & 0.0 & 0.0 & 0.1 & 0.1 & 0.1 & 0.1 & 0.0 & 0.0 \\
\hline Nominal GDP & 377.5 & 393.1 & 413.7 & 447.9 & 476.9 & 510.5 & 544.8 & 581.6 & 621.2 & 662.2 & 705.8 \\
\hline Exports of goods and services & 54.5 & 55.5 & 59.5 & 67.2 & 68.0 & 70.5 & 73.1 & 76.4 & 79.9 & 84.9 & 90.2 \\
\hline Government revenue & 34.6 & 35.4 & 44.6 & 49.9 & 54.4 & 59.1 & 63.6 & 68.1 & 73.1 & 78.2 & 83.6 \\
\hline Debt service 2,4 & 19.8 & 15.9 & 451.9 & 0.5 & 0.7 & 3.5 & 7.3 & 7.1 & 7.4 & 4.5 & 4.8 \\
\hline Net Foreign Assets Central Bank & 66.6 & 60.9 & 62.0 & 72.1 & 74.4 & 75.3 & 76.7 & 78.3 & 80.4 & 85.5 & 90.8 \\
\hline Quota (SDR) & 14.2 & 14.2 & 14.2 & 14.2 & 14.2 & 14.2 & 14.2 & 14.2 & 14.2 & 14.2 & 14.2 \\
\hline
\end{tabular}

Sources: IMF staff estimates and projections.

${ }^{1}$ Data are actual through end-March 2011 and are projected after that

${ }^{2}$ Effective on January 7, 2010 interest on ECF credit outstanding would be zero in 2010 and 2011. It is assumed the ECF interest rate would be at 0.25 percent from 2012 and onward. Charges include net SDR charges and SDR assessments.

${ }^{3}$ A new ECF arrangement of 157.5 percent of quota (SDR 22.365 million) was approved in May 2010, of which 37.5 percent of quota (SDR 5.325 million) was used for early repurchase of outstanding EPCA credit.

${ }^{4}$ Total debt service includes IMF repurchases and repayments.

${ }^{5}$ Guinea-Bissau reached the HIPC completion point in December 2010 and received the remaining HIPC assistance on the stock basis. 


\section{APPENDIX I. SUPPLEMENTARY LETTER OF INTENT}

Bissau, April 29, 2011

Mr. Dominique Strauss-Kahn

Managing Director

International Monetary Fund

Washington, D.C. 20431

U.S.A.

Dear Mr. Strauss-Kahn,

1. This letter of intent and the attached memorandum of economic and financial policies (MEFP) update and supplement our correspondence of November 16, 2010, and describe performance under the government's economic program since then.

2. The government's economic program, supported by a three-year Extended Credit Facility (ECF), is on track. All performance criteria through end-December 2010 have been observed, and structural benchmarks for the second review were met. All quantitative targets for end-December were also met, including the one on social and priority spending (see Tables 1 and 2 of the MEFP).

3. The attached MEFP reflects the government's commitment to the goals of achieving fiscal and external sustainability, reviving economic growth, reducing poverty, and making progress toward the Millennium Development Goals (MDGs). The government believes that the steps taken and economic policies it intends to pursue in the remainder of 2011 are adequate to achieve the targets and objectives under the program (performance criteria and structural benchmarks for the third and fourth review are included in Tables 3 and 4 of the MEFP). However, if necessary, the government will adopt additional measures to achieve these objectives. It will consult with the Fund before adopting such measures or if there are changes in the policies contained in the MEFP, in accordance with the Fund's policies on such consultations.

4. In view of the performance under the ECF-supported program and the government commitment to economic reforms as specified in the MEFP attached, the Government of GuineaBissau requests completion of the second review and the third disbursement under the ECF arrangement of SDR 2.414 million.

5. In line with our commitment to transparency, we request the IMF to publish this letter of intent, the attached MEFP, the Technical Memorandum of Understanding, and the staff report relating to this request.

Sincerely yours,

$/ \mathrm{S} /$

José Mario Vaz

Minister of Finance 


\section{ATTACHMENT I. SUPPLEMENTARY MEMORANDUM OF ECONOMIC AND FINANCIAL POLICIES FOR 2011}

\section{INTRODUCTION}

1. This memorandum updates the MEFP of November 16, 2010, and summarizes the progress achieved since then. The program objectives are unchanged: achieving fiscal and external sustainability, reviving economic growth, and making progress toward poverty alleviation and the Millennium Development Goals (MDGs).

2. Program implementation has taken place against the background of rising import prices for food and fuel prices. The government considers that adjustment is preferable to accommodation in light of the permanent nature of the price shock and the need to secure a full pass-through of price changes over time to encourage consumers and producers to adjust.

\section{Recent Developments and Performance Under the Program}

\section{A. Economic Developments in 2010}

3. Macroeconomic performance improved, owing to a rebound in the price of cashew exports and improved liquidity in the banking system. Strong terms-of-trade gains more than offset a weather-related decline in the cashew harvest. Overall, growth accelerated slightly to 3.5 percent in 2010, up from 3.0 percent in 2009 . Driven by rising import prices for food and fuel, headline inflation accelerated from -1.6 percent in 2009 to 1.1 percent in 2010, still below the WAEMU convergence criterion. The external current account deficit, excluding official transfers, narrowed by about 4 percentage points of GDP.

4. The government adjusted to a shortfall in budget support of 3.2 percent of GDP (or CFAF13.4 billion). Fiscal adjustment was achieved through higher revenue (0.5 percentage points of GDP) and lower spending (2.1 percentage points of GDP), while the government drew down part of its deposits at the BCEAO (0.6 percent of GDP). Higher than programmed revenue reflected tighter controls at customs and revenue administration and more than offset lower disbursements in fishing compensation. The government contained spending through lower outlays on goods and services and underexecution of the public investment program.

\section{B. Performance Under the ECF Program}

5. Despite the shortfall in budget support, fiscal performance has been satisfactory. All performance criteria and indicative targets at end-December 2010 were met (Table 1).

6. All structural benchmarks for the second review have been met. The government made the link from the treasury to customs administration operational; submitted to the 
council of ministers the action plan for public administration reform; and began to use the unified payroll system at the ministries of finance and labor and public administration (Table 2). Progress on other structural measures is detailed in Table 4.

\section{Macroeconomic OUtlook ANd Economic Policies for 2011}

\section{A. Macroeconomic Outlook}

7. The growth outlook is broadly unchanged. Economic growth is expected to accelerate moderately to 4.3 percent in 2011 , driven by rising cashew production, higher cashew prices, and a steady rebuilding of infrastructure, especially roads, electricity, and water. Risks to the outlook would seem to be fairly balanced: downside risks arise from higher-than-expected import prices for food and fuel while there is an upside from higherthan-projected cashew prices in line with recent cashew trading elsewhere.

8. Inflation prospects worsened, reflecting rising import prices of food and fuel. Headline inflation is now projected at 4 percent, above the WAEMU convergence criterion. However, core inflation is expected to remain subdued, and headline inflation should come back within the WAEMU convergence criterion range in 2012, once the one-off effect of rising import prices subsides.

\section{B. Fiscal Policy}

9. The government remains committed to strengthening public finances. Fiscal policy will focus on containing the domestic primary deficit in line with available financing; sustaining efforts to mobilize more revenue; and keeping tight spending controls.

10. The government has secured budget support beyond what has been budgeted for in 2011 and plans to restore public investment under the program. In early March, the government received a new grant from the Angolan government of US\$12 million (about CFAF6 billion or 1.3 percent of GDP) to finance capital spending. The government decided to allocate the new grant received from Angola to finance investments in road and agriculture projects. The government held consultation with the EU under the agreement of Cotonou on March 29, and the EU announced it will condition its resumption of support to Guinea-Bissau on the undertakings by the government in the coming months in several areas, including security sector reform and appointments of military personnel. At the same time, the budget support operations assumed in the program from the World Bank (CFAF3.6 billion) and the African Development Bank (CFAF3.9 billion) are moving forward, with disbursements expected in the second half of the year.

\section{The government will secure a full pass-through of import price changes over} time to encourage consumers and producers to adjust. In early-March, the government reduced duties on fuel products with a view to mitigate the impact on the cost of living, but 
reversed the measure in end-March, after considering the expected permanent nature of the oil price shock and the need to adjust rather than accommodate the shock.

\section{The government will introduce a strong package of measures to reinforce fiscal} revenues this year and prepare the ground to sustain gains in revenue mobilization next year. In addition to restoring the reference price of diesel for customs purpose back to levels that prevailed in early March (CFA 301.68), the government will:

i. adjust the reference price for imported sugar up to market levels by end-April;

ii. adjust the reference price for imported diesel from CFAF301.68 to CFAF373.68 by endJune;

iii. refrain from reducing reference prices for customs purposes;

iv. ensure that the commission for regular adjustment of domestic fuel prices holds monthly meetings to enforce that domestic prices reflect changes in international prices;

v. review the reference prices of rice, flour, diesel, and cashew until the end of the year, with a view to raise them closer to market levels, as part of the 2012 budget; and

vi. reduce or eliminate customs exemptions identified by the current government's review (see below).

\section{Structural Reforms}

13. The government will renew efforts to consolidate gains and sustain its reform agenda as detailed in the MEFP of November 2010 (Tables 3 and 4).

14. The new five-year poverty reduction strategy (PRS) will be submitted to the Council of Ministers by June. The government's medium-term economic reform program supported under the ECF arrangement is in line with the new PRSP. To implement the strategy, the government will coordinate with donors and development partners. Priorities are to identify economic projects in line with the PRS, ensure adequate coordination with donors in support of that strategy, and implement the PIP.

15. In what follows we provide an update of progress on economic reforms and the way forward.

\section{(i) Strengthening Revenue Collection}

16. The government is on track with modernizing and improving customs and tax administration:

a. On customs, the government will (i) finish customizing and testing the ASYCUDA ++ system with the parameters for Guinea-Bissau and train staff with the help of UNCTAD; and (ii) finish the comprehensive review of all custom exemptions to further broaden the customs revenue base. 
b. On tax administration, the government prepared an action plan (with TA from WEST AFRITAC building on the FAD mission of last September) to: (i) revise taxpayer segmentation based on turnover criteria, with thresholds for small and large taxpayers at CFAF10 and 40 million, respectively; (ii) cross-check ASYCUDA data with tax returns for 300 largest importers in 2009-10; and (iii) audit importers underreporting tax liabilities, beginning with largest gaps. A newly set up team at Department of Internal Taxes (DGCI) will lead efforts in this area, with a view to introduce a VAT system by 2013 .

(ii) Modernizing the Public Administration and Creating Space for Priority Spending

17. In March, the Council of Minister approved the medium-term action plan for the reform of the public administration, to make it more modern and efficient. While the reform is gradual and figures will be finalized only after the unification of the payroll system, the plan is to reduce the size of the civil service from over 16,000 in 2010 to about 14,000 by 2015 , with a view to start bringing the ratio from 10 to 6-7 employees per 1000 inhabitants in line with the norm in the WAEMU region.

18. The government has made progress implementing stricter payroll controls and a comprehensive database. The government began using the unified payroll system in the ministries of finance and public function in March, and will start using the system in all ministries by August. The government has started implementing the personnel management (SIGRHAP), which is expected to be operational by end-August.

19. The government is working with partners to implement defense and security sector reform (SSR). Following a decision by the European Union (EU) not to deploy a new mission to monitor the implementation phase of the SSR, the government strengthened coordination with the United Nations Integrated Peace-Building Office in Guinea-Bissau (UNIOGBIS) and regional and bilateral partners, including ECOWAS and the Community of Portuguese Language Countries (CPLP), to prepare a plan to ensure adequate financing of the reform, especially of the pension fund. The UN announced in February its Priority Plan for Guinea-Bissau with an allocation of US\$16 million for civilian-led aspects of the security sector reform. In March, the Council of Ministers approved the ECOWAS-CPLP road map for the SSR.

(iii) Removing Impediments to Private Sector Development

20. The government will intensify efforts to prepare an action plan that removes impediments to private sector development and improves the ease of doing business in Guinea-Bissau. The government will also cooperate with the World Bank to prepare a plan to manage the country's natural resources by December. 


\section{(iv) Reinforcing Debt Management and Arrears Clearance}

21. In the wake of the HIPC completion point in December, the government will seek to reinforce its debt management. With the assistance of UNCTAD, the Public Debt Office plans to hire and train new staff, start producing quarterly electronic reports on public debt by June 2011, and start using SYGADE by December to record, monitor, and manage all public debt. UNCTAD has begun providing training for government staff on SYGADE.

22. The government has requested a deferral of payment and better terms on debts owed to the Central Bank of West African States (BCEAO). The renegotiation of the debt owed to the BCEAO (about CFAF90 billion or 20 percent of GDP) has been suspended until a new BCEAO governor is appointed. The debt with the BCEAO stems from several sources: a required capital contribution to join the WAEMU; debt from the BCEAO's repayment, on behalf of the government, of previous IMF credit to Guinea-Bissau; and the on-lending of the SDR allocation.

23. The government will continue with its medium-to-long term arrears clearance strategy of making annual payments consistent with available resources. A team of independent auditors issued a preliminary audit report for 2000-07 arrears. The government is verifying the audit with a view to incorporate verified arrears into its arrears strategy.

\section{Financial Sector and Monetary Policies}

24. Monetary policy will continue to be pursued within the WAEMU framework. This arrangement has enabled Guinea-Bissau to contain inflation and maintain a stable exchange rate. As the rise in import prices for fuel and food wears off, domestic inflation is expected to return within the WAEMU target by 2012.

\section{E. Post-HIPC Completion Point Work}

25. The government is taking steps to implement the HIPC completion point. It has asked all creditors to confirm their intention to provide HIPC and topping up debt relief. The government received topping up assurances from Portugal, thus reaching the 80 percent commitment threshold necessary for the World Bank to proceed with topping up and MDRI assistance. Negotiations with the Paris Club are now expected to take place in May.

\section{F. Capacity Building and Technical Assistance}

26. The government has identified technical assistance priorities in revenue administration, tax policy, and debt management.

- Revenue administration: In 2010, a FAD technical assistance (TA) mission performed an assessment and made recommendations for the government to strengthen revenue administration. The government will implement these recommendations with technical 
assistance from the regional AFRITAC and will seek further technical assistance from FAD.

- Tax policy: The government plans to introduce a value-added tax (VAT) by 2013, in line with other WAEMU countries. Initial steps and technical assistance are needed to support the design, preparation, and implementation of the VAT.

- Debt management: Although its external debt outlook has improved significantly since debt relief, debt management has emerged as an urgent new priority. The government will seek technical assistance to strengthen public debt management, including the public debt unit at the Ministry of Finance, so that it can record and manage all public debt and produce quarterly reports on public debt.

\section{G. Program Monitoring}

27. The second year of the program covers the 12 months from January 1, 2011, to December 31, 2011. The program will be monitored using quarterly quantitative indicators and structural benchmarks, as well as semiannual reviews and the quantitative performance criteria presented in Table 3 . Tables 4 and 5 contain a list of structural measures identified by the government, including the structural benchmarks for the third and fourth review. The definitions of quantitative performance criteria and benchmarks are in the attached TMU. The government expects the third review to take place in December 2011 and the fourth review is expected to take place in May 2012. 
Table 1. Guinea-Bissau: Quantitative Indicators for the ECF Program for 2010

Quarterly Targets ${ }^{1}$

(Cumulative, CFAF millions)

\begin{tabular}{|c|c|c|c|c|c|c|c|c|}
\hline & \multicolumn{2}{|c|}{ End-March } & \multicolumn{2}{|c|}{ End-June } & \multicolumn{2}{|c|}{ End-Sept. } & \multicolumn{2}{|c|}{ End-Dec. } \\
\hline & Prog. $^{2}$ & Actual & Prog. $^{2}$ & Actual & Prog. $^{2}$ & Actual & Prog. $^{2}$ & Prel. \\
\hline \multicolumn{9}{|l|}{ Performance criteria ${ }^{3}$} \\
\hline 1. Domestic financing of the budget & 10,618 & 5,975 & 8,627 & 302 & 8,736 & 4,308 & 12,231 & 3,409 \\
\hline 2. New domestic arrears & 0 & 0 & 0 & 0 & 0 & 0 & 0 & 0 \\
\hline 3. External nonconcessional public borrowing, maturity $>1$ year & 0 & 0 & 0 & 0 & 0 & 0 & 0 & 0 \\
\hline 4. External short term public borrowing & 0 & 0 & 0 & 0 & 0 & 0 & 0 & 0 \\
\hline 5. New external payment arrears & 0 & 0 & 0 & 0 & 0 & 0 & 0 & 0 \\
\hline \multicolumn{9}{|l|}{ Indicative targets } \\
\hline 6. Government tax revenue & 5,420 & 6,391 & 15,148 & 17,538 & 24,218 & 25,394 & 30,101 & 33,086 \\
\hline 7. Domestic primary balance (commitment basis) & $-7,789$ & $-2,401$ & $-8,386$ & 1,048 & $-11,030$ & $-1,352$ & $-17,938$ & $-4,865$ \\
\hline 8. Nonregularized expenditure (DNTs) & 200 & 0 & 200 & 0 & 200 & 0 & 200 & 0 \\
\hline 9. Social and priority spending & 4,062 & 1,989 & 8,123 & 4,223 & 12,185 & 6,818 & $8,647^{4}$ & 9,152 \\
\hline
\end{tabular}

${ }^{1}$ Cumulative from January 1. The definition of the aggregates and adjustors is provided in the Technical Memorandum of Understanding (TMU).

${ }^{2}$ Adjusted target under the program.

${ }^{3}$ Performance criteria (PC) are defined for June and December. March and September are indicative. All performance criteria (PC) are ceillings and PC 3,4 , and 5 apply continously.

${ }^{4}$ Indicative target for social and priority spending was revised compared to program (IMF Country Report No. 10/379) from CFAF 16,246 to CFAF 8,647. 
Table 2. Guinea-Bissau: Structural Benchmarks Under the ECF Second Review

\begin{tabular}{|c|c|c|c|c|c|c|}
\hline Category & & Structural Benchmarks & $\begin{array}{c}\text { Macro } \\
\text { Rationale }\end{array}$ & $\begin{array}{l}\text { Actual } \\
\text { Delivery } \\
\text { Date }\end{array}$ & Ministry & $\begin{array}{l}\text { Status of } \\
\text { Implementa- } \\
\text { tion }\end{array}$ \\
\hline $\begin{array}{l}\text { Fiscal } \\
\text { management }\end{array}$ & 4 & $\begin{array}{l}\text { Establish an operational link from } \\
\text { budget/treasury under the SIGFIP to } \\
\text { the customs and tax administration. }\end{array}$ & $\begin{array}{l}\text { Greater control } \\
\text { in budget } \\
\text { execution }\end{array}$ & March 2011 & $\mathrm{MoF}^{1}$ & Met \\
\hline $\begin{array}{l}\text { Expenditure } \\
\text { reforms }\end{array}$ & 5 & $\begin{array}{l}\text { Submit to the Council of Ministers an } \\
\text { action plan for public administration } \\
\text { reform, with a medium-term schedule } \\
\text { to downsize the civil service through } \\
\text { retirement and removing redundant } \\
\text { workers beginning in } 2011 \text {. }\end{array}$ & $\begin{array}{l}\text { To improve the } \\
\text { efficiency of civil } \\
\text { service }\end{array}$ & Jan 2011 & $\begin{array}{l}\text { MoF } \\
\text { MinLaPS }\end{array}$ & Met \\
\hline
\end{tabular}

${ }^{1}$ Ministry of Finance

${ }^{2}$ Ministry of Labor and Public Administration 
Table 3. Guinea-Bissau: Quantitative Indicators for the ECF Program for 2011 Quarterly Targets ${ }^{1}$

(Cumulative, CFAF millions)

\begin{tabular}{|c|c|c|c|}
\hline End-March & End-June & End-Sept. & End-Dec. \\
\hline Prog. & Prog. & Prog. & Prog. \\
\hline
\end{tabular}

\section{Performance criteria ${ }^{2}$}

1. Domestic financing of the budget

2. New domestic arrears

3. External nonconcessional public borrowing, maturity $>1$ year

4. External short term public borrowing

5. New external payment arrears

1,431
0

$$
-520
$$

$-1,954$

0

0

$0 \quad 0$

0

0

0

Indicative targets ${ }^{3}$

6. Government tax revenue

7. Domestic primary balance (commitment basis)

8. Nonregularized expenditure (DNTs)

${ }^{1}$ Cumulative from January 1 . The definition of the aggregates and adjustors is provided in the Technical Memorandum of Understanding (TMU).

${ }^{2}$ Performance criteria (PC) are defined for June and December. March and September are indicative. All PC are ceillings and PC 3,4 , and 5 apply continously.

${ }^{3}$ The indicative targets 6, 7 and 9 were revised compared to program (IMF Country Report No. 10/379) to reflect the higher revenue base from 2010 and the additional budget support. 
Table 4. Guinea-Bissau: Structural Benchmarks Under the ECF

January 1, 2011-December 31, 2011

\begin{tabular}{|c|c|c|c|c|c|}
\hline Category & & $\begin{array}{l}\text { Structural Benchmarks } \\
\qquad \text { Third Review }\end{array}$ & Macro Rationale & Ministry & $\begin{array}{c}\text { Status of } \\
\text { implementation }\end{array}$ \\
\hline \multirow{3}{*}{$\begin{array}{l}\text { Tax reform and } \\
\text { strengthening } \\
\text { revenue } \\
\text { collection }\end{array}$} & 1 & $\begin{array}{l}\text { Customize and test ASYCUDA ++, } \\
\text { and provide training to staff. }\end{array}$ & $\begin{array}{l}\text { To strengthen revenue } \\
\text { collection }\end{array}$ & $\mathrm{MoF}^{1}$ & In progress \\
\hline & 2 & $\begin{array}{l}\text { Conduct a comprehensive review } \\
\text { of all customs exemptions to } \\
\text { broaden the customs revenue base } \\
\text { and submit report to Council of } \\
\text { Ministers. }\end{array}$ & $\begin{array}{l}\text { To strengthen revenue } \\
\text { collection }\end{array}$ & MoF & In progress \\
\hline & 3 & $\begin{array}{l}\text { Cross-check ASYCUDA data with } \\
\text { tax returns for } 300 \text { largest } \\
\text { importers in 2009-10. }\end{array}$ & $\begin{array}{l}\text { To strengthen revenue } \\
\text { collection }\end{array}$ & MoF & In progress \\
\hline $\begin{array}{l}\text { Expenditure } \\
\text { reforms }\end{array}$ & 4 & $\begin{array}{l}\text { Extend the computerized and } \\
\text { unified payroll and personnel } \\
\text { management system to all } \\
\text { ministries. }\end{array}$ & $\begin{array}{l}\text { To modernize public } \\
\text { administration }\end{array}$ & $\begin{array}{l}\text { MoF } \\
\text { MinLaPS }^{2}\end{array}$ & In progress \\
\hline $\begin{array}{l}\text { Business } \\
\text { environment }\end{array}$ & 5 & $\begin{array}{l}\text { Prepare an action plan to identify } \\
\text { and remove further impediments to } \\
\text { private sector development and } \\
\text { improve the ease of doing } \\
\text { business in Guinea-Bissau. }\end{array}$ & $\begin{array}{l}\text { Improve the ease of } \\
\text { doing business }\end{array}$ & MinEcon $^{3}$ & In progress \\
\hline $\begin{array}{l}\text { Public debt } \\
\text { management }\end{array}$ & 6 & $\begin{array}{l}\text { Start producing, on a quarterly } \\
\text { basis, electronic reports on public } \\
\text { debt (external and domestic). }\end{array}$ & Improve transparency & $\mathrm{MoF}$ & In progress \\
\hline
\end{tabular}

\section{Fourth Review}

\begin{tabular}{|c|c|c|c|c|}
\hline $\begin{array}{l}\text { Public debt } \\
\text { management }\end{array}$ & 1 & $\begin{array}{l}\text { Use SYGADE to record, monitor, } \\
\text { and manage all public debt } \\
\text { (external and domestic) }\end{array}$ & $\begin{array}{l}\text { Improve debt } \\
\text { management }\end{array}$ & MoF \\
\hline \multirow{2}{*}{$\begin{array}{l}\text { Tax reform and } \\
\text { strengthening } \\
\text { revenue } \\
\text { collection }\end{array}$} & 2 & $\begin{array}{l}\text { Audit those importers } \\
\text { underreporting their tax liabilities, } \\
\text { beginning with largest gaps. }\end{array}$ & $\begin{array}{l}\text { To strengthen revenue } \\
\text { collection }\end{array}$ & MoF \\
\hline & 3 & $\begin{array}{l}\text { Review the legal framework that } \\
\text { regulates revenue sharing } \\
\text { agreements ("restitutions"). }\end{array}$ & $\begin{array}{l}\text { To strengthen revenue } \\
\text { collection }\end{array}$ & MoF \\
\hline $\begin{array}{l}\text { Business } \\
\text { environment }\end{array}$ & 4 & $\begin{array}{l}\text { Prepare a plan to manage the } \\
\text { country's natural resources. }\end{array}$ & $\begin{array}{l}\text { Improve the ease of } \\
\text { doing business }\end{array}$ & MinEcon \\
\hline
\end{tabular}


Table 5. Guinea-Bissau: Structural Measures for 2011

\begin{tabular}{|c|c|c|c|}
\hline Category & & Measures & $\begin{array}{l}\text { Delivery } \\
\text { Date }\end{array}$ \\
\hline \multirow{15}{*}{$\begin{array}{l}\text { Tax reform \& } \\
\text { strengthening } \\
\text { revenue } \\
\text { collection }\end{array}$} & 1 & Raise reference price for cashew exports to US $\$ 750$ per ton. & 2011 \\
\hline & 2 & $\begin{array}{l}\text { Raise the reference price for staple imports (rice to CFAF6000, sugar to CFAF8500, and } \\
\text { flour to CFAF8500). }\end{array}$ & 2011 \\
\hline & 3 & $\begin{array}{l}\text { Increase the tax rate on advance payment of the profit tax collected at customs } \\
\text { (anticipation of industrial contribution) from } 3 \text { to } 5 \text { percent for the informal sector. }\end{array}$ & 2011 \\
\hline & 4 & Reintroduce seals on alcoholic beverages and tobacco. & Jan 2011 \\
\hline & 5 & $\begin{array}{l}\text { Customize and test the system of ASYCUDA ++ with the parameters for Guinea-Bissau } \\
\text { (including the specific exemptions and tariffs), and train staff. }\end{array}$ & Aug 2011 \\
\hline & 6 & Restore the reference price for diesel for customs purpose back to early-March levels. & Apr 2011 \\
\hline & 7 & Adjust the reference price for imported sugar up to market levels. & Apr 2011 \\
\hline & 8 & Adjust the reference price for imported diesel from CFAF301.7 to CFAF373.0 & Jun 2011 \\
\hline & 9 & $\begin{array}{l}\text { Review price of rice, flour, diesel, and cashew, with a view to raise the reference price for } \\
\text { these products to market levels by December 2011, to be incorporated in the } 2012 \\
\text { budget. }\end{array}$ & Dec 2011 \\
\hline & 10 & $\begin{array}{l}\text { Conduct a comprehensive review of all customs exemptions, with a view to reduce or } \\
\text { eliminate identified exemptions. }\end{array}$ & Jun 2011 \\
\hline & 11 & $\begin{array}{l}\text { Revise taxpayer segmentation based on turnover criteria, with threshold for small and } \\
\text { large taxpayers at CFAF10 and } 40 \text { million, respectively. }\end{array}$ & Jun 2011 \\
\hline & 12 & Cross-check ASYCUDA data with tax returns for 300 largest importers in 2009-10. & Jun 2011 \\
\hline & 13 & Audit importers underreporting tax liabilities, beginning with largest gaps. & Dec 2011 \\
\hline & 14 & Increase to 90 percent large taxpayers filing rate for the general sales tax. & 2011 \\
\hline & 15 & Continue to reinforce the large taxpayer unit in the Ministry of Finance. & 2011 \\
\hline \multirow[t]{7}{*}{$\begin{array}{l}\text { Fiscal } \\
\text { management }\end{array}$} & 16 & $\begin{array}{l}\text { Review the legal framework that regulates revenue sharing agreements ("restitutions") to } \\
\text { increase the share of nonearmarked revenues in the budget. }\end{array}$ & Dec 2011 \\
\hline & 17 & Seek technical assistance to reinforce budget preparation procedures. & Jun 2012 \\
\hline & 18 & Prepare and regularly updates the treasury plan for the 2011 budget. & 2011 \\
\hline & 19 & $\begin{array}{l}\text { Establish an operational link form budget/treasury under the SIGFIP to the customs and } \\
\text { tax administration. }\end{array}$ & Mar 2011 \\
\hline & & Continue publishing quarterly budget execution reports. & 2011 \\
\hline & & Modify the organic laws for the Audit Court. & 2011 \\
\hline & 22 & Submit to Parliament the budgetary execution accounts for 2010 . & Sep 2011 \\
\hline
\end{tabular}




\begin{tabular}{|c|c|c|c|}
\hline \multirow[t]{3}{*}{$\begin{array}{l}\text { Civil service } \\
\text { reform }\end{array}$} & 23 & $\begin{array}{l}\text { Submit to the Council of Ministers an action plan for public administration reform, with a } \\
\text { medium-term schedule to downsize the civil service through retirement and removing } \\
\text { redundant workers beginning in } 2011 \text {. }\end{array}$ & Jan 2011 \\
\hline & 24 & $\begin{array}{l}\text { Start using the computerized and unified payroll and personnel management system in } \\
\text { the Ministries of Finance and Public Service. }\end{array}$ & Jan 2011 \\
\hline & 25 & $\begin{array}{l}\text { Extend the computerized and unified payroll and personnel management system to all } \\
\text { ministries. }\end{array}$ & Aug 2011 \\
\hline $\begin{array}{l}\text { Defense and } \\
\text { security sector } \\
\text { reform }\end{array}$ & 26 & $\begin{array}{l}\text { Prepare an operational plan with a list of potential retirees and projects for the } \\
\text { construction of military and security installations. }\end{array}$ & 2011 \\
\hline $\begin{array}{l}\text { Arrears } \\
\text { clearance }\end{array}$ & 27 & Complete the audit of $2000-07$ private sector arrears. & 2011 \\
\hline \multirow{3}{*}{$\begin{array}{l}\text { Business } \\
\text { environment }\end{array}$} & 28 & Adopt the implementing regulations of the new investment code. & 2011 \\
\hline & 29 & $\begin{array}{l}\text { Prepare an action plan to further identify and remove impediments to private sector } \\
\text { development and improve the ease of doing business in Guinea-Bissau. }\end{array}$ & Aug 2011 \\
\hline & 30 & Prepare a plan to manage the county's natural resources. & Dec 2011 \\
\hline Social services & 31 & Adopt a new five-year poverty reduction strategy (PRS). & Jun 2011 \\
\hline \multirow{3}{*}{$\begin{array}{l}\text { Public debt } \\
\text { management }\end{array}$} & 32 & Strengthen the unit of public debt management. & 2011 \\
\hline & 33 & $\begin{array}{l}\text { Produce quarterly electronic reports on public debt no later than three months after each } \\
\text { quarter, in a manner consistent with the BCEAO reporting requirements. }\end{array}$ & Jun 2011 \\
\hline & 34 & Use SYGADE to record, monitor, and manage all public debt. & Dec 2011 \\
\hline
\end{tabular}




\title{
Attachment II. Technical Memorandum OF Understanding
}

\author{
Bissau
}

April 29, 2011

1. This memorandum updates the Technical Memorandum of Understanding of November 16, 2010 and describes the definitions of the quantitative and structural performance criteria, indicative targets, and structural benchmarks to monitor the program supported by an arrangement under the Extended Credit Facility (ECF). It also specifies the agreed periodicity and deadlines for transmission of data to IMF staff for program monitoring.

\section{Quantitative Indicators and Adjustors}

\section{A. Quantitative Indicators}

2. The quantitative indicators are as follows:

a. Cumulative floors on government tax revenue

b. Cumulative ceilings on the domestic primary fiscal deficit (on a commitment basis)

c. Cumulative ceiling on the amount of nonregularized expenditures (DNTs).

d. Cumulative floor on social and other priority spending

e. Cumulative ceilings on the change in net domestic financing of the budget

f. Cumulative ceiling on new domestic arrears of the government, including wage arrears

g. Cumulative ceilings on new nonconcessional external debt contracted or guaranteed by the government

h. Cumulative ceiling on new external short term debt

i. Cumulative ceiling on new external payment arrears.

Quantitative indicators are set for end-March, end-June, end-September, and end-December. Quantitative performance criteria are proposed for end-June and end-December 2011 for indicators (e) through (i). Indicators for new nonconcessional external debt, new external short-term debt, and new external payment arrears are continuous.

\section{Definitions and computation}

3. Government. Unless otherwise indicated, "Government" means the central administration of the Republic of Guinea-Bissau and does not include any local 
administration, the central bank, or any other public or government-owned entity with autonomous legal personality not included in the government flow-of-funds table (TOFE).

4. The targeted floor on government tax revenues includes direct and indirect taxes, as well as recovery of tax arrears and additional revenue efforts (Table 1).

Table 1. Quarterly Floors for Government Tax Revenues, 2011

(Cumulative, CFAF millions)

\begin{tabular}{|c|c|c|c|c|}
\hline & March & June & Sept. & Dec. \\
\hline & Prog. & Prog. & Prog. & Prog. \\
\hline Tax revenues & 6,437 & 17,988 & 30,506 & 37,917 \\
\hline
\end{tabular}

5. The domestic primary fiscal deficit on a commitment basis is calculated as the difference between government revenue and domestic primary expenditure on a commitment basis (Table 2). Government revenue includes all tax and nontax receipts and excludes external grants. Domestic primary expenditure consists of current expenditure plus domestically financed capital expenditure, excluding all interest payments. Government commitments include all expenditure for which commitment vouchers have been approved by the Ministry of Finance; automatic expenditure (such as wages and salaries, pensions, utilities, and other expenditure for which payment is centralized); and expenditure by means of offsetting operations.

Table 2. Quarterly Domestic Primary Balance, New Domestic Arrears, and Nonregularized Expenditures, 2011 (Cumulative, CFAF millions)

\begin{tabular}{|c|c|c|c|c|}
\hline & March & June & Sept. & Dec. \\
\hline & Prog. & Prog. & Prog. & Prog. \\
\hline Total domestic primary deficit & $-3,597$ & $-5,842$ & $-5,911$ & $-12,087$ \\
\hline Revenue & 7,813 & 21,253 & 39,449 & 49,860 \\
\hline Domestic primary expenditure & 11,410 & 27,095 & 45,361 & 61,947 \\
\hline New domestic arrears & 0 & 0 & 0 & 0 \\
\hline Nonregularized expenditures (DNTs) & 200 & 200 & 200 & 200 \\
\hline
\end{tabular}

6. New domestic arrears of the government are defined as accounts payable (rest-apayer) accumulated during the year, still outstanding one month after the quarter for wages and salaries (including pensions), and three months after for goods and services and transfers, at end-March, end-June, end-September, and end-December 2011.

7. Nonregularized expenditures are defined as any treasury outlay not classified in the expenditure tables presented by the National Budget Directorate.

8. Net domestic financing consists of bank and nonbank financing (Table 3 ). Bank financing consists of net changes in the balances of the treasury accounts at the BCEAO (excluding net disbursement from the IMF) and commercial banks (excluding balances in those accounts that are not available for budget financing, such as accounts held under 
double signature arrangements with donors) and in the outstanding amounts of loans, including T-bills, from the BCEAO and commercial banks, local and regional. Nonbank financing encompasses privatization receipts and any other financial debt held outside the banking system other than new domestic arrears.

Table 3. Domestic Financing by Quarter, 2011

(Cumulative, CFAF millions)

\begin{tabular}{crrrr}
\hline & March & June & Sept. & Dec. \\
\cline { 2 - 5 } & Prog. & Prog. & Prog. & Prog. \\
\hline Domestic financing & 1,431 & -520 & $-1,954$ & 0 \\
Bank financing & 1,431 & -520 & $-1,954$ & 0 \\
BCEAO & 1,431 & -520 & $-1,954$ & 0 \\
Commercial banks (including regional) & 0 & 0 & 0 & 0 \\
Regional commercial banks and Treasury bills & 0 & 0 & 0 \\
Nonbank financing & & 0 & 0 & 0 \\
\hline
\end{tabular}

\section{Contracting or Guaranteeing of New Nonconcessional External Debt by the Government.}

9. Definition of debt. For the purposes of the relevant performance criteria, the definition of debt is set out in Executive Board Decision No.6230-(79/140), Point 9, as revised on August 31, 2009 (Decision No. 14416-(09/91)).

a. the term "debt" will be understood to mean a current, i.e., not contingent, liability, created under a contractual arrangement through the provision of value in the form of assets (including currency) or services, and which requires the obligor to make one or more payments in the form of assets (including currency) or services, at some future point(s) in time; these payments will discharge the principal and/or interest liabilities incurred under the contract. Debts can take a number of forms, the primary ones being as follows:

i. loans, i.e., advances of money to the obligor by the lender made on the basis of an undertaking that the obligor will repay the funds in the future (including deposits, bonds, debentures, commercial loans and buyers' credits) and temporary exchanges of assets that are equivalent to fully collateralized loans under which the obligor is required to repay the funds, and usually pay interest, by repurchasing the collateral from the buyer in the future (such as repurchase agreements and official swap arrangements);

ii. suppliers' credits, i.e., contracts where the supplier permits the obligor to defer payments until sometime after the date on which the goods are delivered or services are provided; and

iii. leases, i.e., arrangements under which property is provided which the lessee has the right to use for one or more specified period(s) of time that are usually 
shorter than the total expected service life of the property, while the lessor retains the title to the property. For the purpose of the guideline, the debt is the present value (at the inception of the lease) of all lease payments expected to be made during the period of the agreement excluding those payments that cover the operation, repair or maintenance of the property.

b. Under the definition of debt above, arrears, penalties, and judicially awarded damages arising from the failure to make payment under a contractual obligation that constitutes debt are debt. Failure to make payment on an obligation that is not considered debt under this definition (e.g., payment on delivery) will not give rise to debt.

10. Debt guarantees. For the purposes of the relevant performance criteria, the guarantee of a debt arises from any explicit legal obligation of the government to service a debt in the event of nonpayment by the debtor (involving payments in cash or in kind).

11. Debt concessionality. For the purposes of the relevant performance criteria, a debt is considered concessional if it includes a grant element of at least 50 percent; ${ }^{1}$ the grant element is the difference between the present value (PV) of debt and its nominal value, expressed as a percentage of the nominal value of the debt. The PV of debt at the time of its contracting is calculated by discounting the future stream of payments of debt service due on this debt. ${ }^{2}$ The discount rates used for this purpose are the currency specific commercial interest reference rates (CIRRs), published by OECD. ${ }^{3}$ For debt with a maturity of at least 15 years, the ten-year-average CIRR is used to calculate the PV of debt and, hence, its grant element. For debt with a maturity of less than 15 years, the six-month average CIRR is used. The margins for differing repayment periods ( 0.75 percent for repayment periods of less than 15 years, 1 percent for 15 to 19 years, 1.15 percent for 20 to 29 years, and 1.25 percent for 30 years or more) are added to the ten-year and six-month CIRR averages.

12. External debt. For the purposes of the relevant performance criteria, external debt is defined as debt borrowed or serviced in a currency other than the CFA franc. This definition also applies to debt among WAEMU countries.

13. Debt-related performance criteria. The relevant performance criteria apply to the contracting and guaranteeing of new nonconcessional external debt by the government. The criteria apply to debt and commitments contracted or guaranteed for which value has not yet

\footnotetext{
${ }^{1}$ The following reference on the IMF website creates a link to a tool that allows for the calculation of the grant element of a broad range of financing packages: http://www.imf.org/external/np/pdr/conc/calculator.

${ }^{2}$ The calculation of concessionality will take into account all aspects of the debt agreement, including maturity, grace period, payment schedule, upfront commissions, and management fees.

${ }^{3}$ For debts in foreign currencies for which the OECD does not calculate a CIRR, calculation of the grant element should be based on the composite CIRR (weighted average) of the currencies in the SDR basket.
} 
been received. The criteria also apply to private debt for which official guarantees have been extended and which, therefore, constitute a contingent liability of the government. The performance criteria are measured on a cumulative basis from the time of approval of the ECF by the Executive Board. PCs will be monitored on a continuous basis. No adjuster will be applied to these criteria.

\section{Ceiling on short-term external debt newly contracted or guaranteed by the} government. Short-term external debt is external debt with the contractual term of less than one year. Debt-relief operations and treasury bills issued in CFA francs on the WAEMU regional market are excluded from this performance criterion. In the context of the program, the government and public enterprises will not contract, or guarantee, short-term external debt. This performance criterion is monitored on a continuous basis.

15. Ceiling on new external payment arrears. External payment arrears are defined as external payments due but not paid on the due date. In cases where a creditor has granted a grace period after the contractual due date, arrears are incurred following the expiration of the grace period. Under the program, the government undertakes not to accumulate arrears on its external debt, except those arising from government debt that is being renegotiated with creditors, including non-Paris Club bilateral creditors. This performance criterion is monitored on a continuous basis.

16. Reporting requirements. The government will report any new external borrowing and its terms to Fund staff as soon as external debt is contracted or guaranteed by the government, but no later than within two weeks of such external debt being contracted or guaranteed.

17. Social and other priority spending is defined as total current expenditures in the education, health, and agricultural sector, and domestically financed capital spending (Table 4).

Table 4. Social and Priority Spending ${ }^{1}$ by Quarter, 2011

(Cumulative, CFAF millions)

\begin{tabular}{lrrrr}
\hline & March & June & Sept. & Dec. \\
\cline { 2 - 5 } & Prog. & Prog. & Prog. & Prog. \\
\hline Social and other priority spending & & & & \\
$\quad$ Education & 2,932 & 5,864 & 8,795 & 12,227 \\
Health & 1,649 & 3,299 & 4,948 & 6,898 \\
Agriculture & 897 & 1,794 & 2,691 & 3,788 \\
Infrastructure & 264 & 527 & 791 & 1,055 \\
& 122 & 244 & 365 & 487 \\
\hline
\end{tabular}

${ }^{1}$ Current and domestically financed capital spending. 


\section{B. Adjustors}

\section{The following adjustors will be in effect:}

a. The ceiling on the domestic primary deficit (on a commitment basis) will be increased in case of lower than programmed disbursement of fishing compensation, by the amount of the shortfall up to a maximum of CFAF3 billion. The program assumes the following amounts of fishing compensation (cumulative from January 1, 2011): zero by endMarch; zero by end-June; by end-September CFAF5 billion; and by end-December CFAF5 billion. ${ }^{4}$

b. The ceiling on domestic financing will be increased for any shortfall in external budget support, and fishing compensation, by the amount of the shortfall up to a maximum of CFAF3.5 billion. The program assumes the following amounts of external budget support, and fishing compensation (cumulative from January 1, 2011): zero by end-March; zero by end-June; by end-September CFAF8.9 billion; and by end-December CFAF12.5 billion.

c. The ceiling on domestic financing for March, June, and September will be increased for payment of previous years arrears in excess of programmed amounts up to a maximum of CFAF1.85 billion. The program assumes the following arrears payments from previous years (cumulative from January 1, 2011): zero by end-March; zero by end-June CFAF; zero by end-September; and by end-December CFAF1.85 billion.

\footnotetext{
${ }^{4}$ For this TMU, the CFAF/euro exchange rate is 655.956 and the CFAF/US\$ exchange rate is 524 .
} 


\title{
INTERNATIONAL MONETARY FUND
}

\author{
GUINEA-BISSAU
}

Second Review Under the Three-Year Arrangement Under the Extended Credit Facility and Financing Assurances Review-Informational Annex

\author{
Prepared by the African Department \\ (In consultation with other Departments)
}

May 10, 2011

Contents

Page

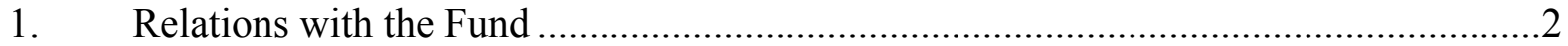

2. Relations with the World Bank Group...................................................................10

3. Relations with the African Development Bank …….................................................12

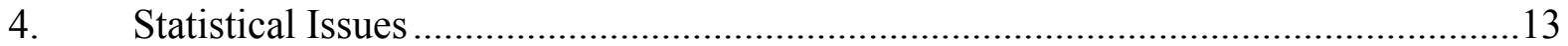




\section{Guinea-Bissau: Relations with the Fund \\ (As of March 31, 2011)}

I. Membership Status: Joined: March 24, 1977;

Article VIII

\section{General Resources Account:}

Quota

Fund holdings of currency

Reserve Tranche Position

Holdings Exchange Rate

\section{SDR Department:}

Net cumulative allocation

Holdings

IV. Outstanding Purchases and Loans:

ECF Arrangements
SDR Million

14.20

14.06

0.14

SDR Million

13.60

12.39

SDR Million

2.41
\%Quota

100.00

99.05

\%Allocation

100.00

91.08

\%Quota

17.00

\section{Latest Financial Arrangements:}

\begin{tabular}{llcrr} 
Type & $\begin{array}{c}\text { Date of } \\
\text { Arrangement }\end{array}$ & $\begin{array}{c}\text { Expiration } \\
\text { Date }\end{array}$ & $\begin{array}{c}\text { Amount Approved } \\
\text { (SDR Million) }\end{array}$ & $\begin{array}{c}\text { Amount Drawn } \\
\text { (SDR Million) }\end{array}$ \\
\cline { 3 - 5 } ECF & May 07, 2010 & May 06, 2013 & 22.37 & 7.88 \\
ECF $^{1 /}$ & Dec 15, 2000 & Dec 14, 2003 & 14.20 & 5.08 \\
ECF $^{1 /}$ & Jan 18, 1995 & Jul 24, 1998 & 10.50 & 10.50
\end{tabular}

${ }^{1 /}$ Formerly PRGF

\section{Projected Payments to Fund ${ }^{2 /}$}

(SDR Million; based on existing use of resources and present holdings of SDRs):

\begin{tabular}{|c|c|c|c|c|c|}
\hline & 2011 & 2012 & 2013 & 2014 & 2015 \\
\hline Principal & 0.00 & 0.00 & 0.00 & 0.00 & 0.00 \\
\hline Charges/Interest & 0.00 & 0.01 & 0.01 & 0.01 & 0.01 \\
\hline Total & 0.00 & 0.01 & 0.01 & 0.01 & 0.01 \\
\hline
\end{tabular}

${ }^{2 /}$ When a member has overdue financial obligations outstanding for more than three months, the amount of such arrears will be shown in this section.

\section{Implementation of HIPC Initiative:}

I. Commitment of HIPC assistance

Enhanced

Decision point date

Framework

Assistance committed

by all creditors (US\$ Million) ${ }^{1 /}$

Dec 2000

Of which: IMF assistance (US\$ million)

(SDR equivalent in millions) 
II. Disbursement of IMF assistance (SDR Million)

$\begin{array}{ll}\text { Assistance disbursed to the member } & 9.20\end{array}$

$\begin{array}{ll}\text { Interim assistance } & 1.56\end{array}$

$\begin{array}{ll}\text { Completion point balance } & 7.64\end{array}$

Additional disbursement of interest income 2/ $\quad 0.23$

$\begin{array}{lr}\text { Total disbursements } & 9.43\end{array}$

${ }^{1 /}$ Assistance committed under the original framework is expressed in net present value (NPV) terms at the completion point, and assistance committed under the enhanced framework is expressed in NPV terms at the decision point. Hence these two amounts cannot be added.

${ }^{2 /}$ Under the enhanced framework, an additional disbursement is made at the completion point corresponding to interest income earned on the amount committed at the decision point but not disbursed during the interim period.

VIII. Implementation of Multilateral Debt Relief Initiative (MDRI): Not Applicable

\section{Implementation of Post-Catastrophe Debt Relief (PCDR): Not Applicable}

\section{Safeguards Assessments:}

The Central Bank of West African States (BCEAO) is a common central bank of the countries of the West African Economic and Monetary Union (WAMU). The most recent safeguards assessment of the BCEAO was completed on March 1, 2010. The 2010 update assessment found that the BCEAO continues to have controls in place at the operational level. The overall governance framework needed nonetheless to be strengthened by the addition of an audit committee to ensure that the Board of Directors exercises appropriate oversight over the control structure, including the audit mechanisms and financial statements. The Institutional Reform of the WAMU and the BCEAO completed after the approval of the safeguards report stipulated creation of the Audit Committee, which should now start working. Efforts to implement fully the International Financial Reporting Standards reporting framework should also be pursued.

\section{Exchange System and Exchange Rate Arrangement}

Guinea Bissau accepted the obligations of Article VIII, Sections 2, 3, and 4 with effect from January 1, 1997. It joined the West African Economic and Monetary Union (WAEMU) in 1997, and has no separate legal tender. The exchange system common to all members of the union is free from multiple currency practices and exchange restrictions on the making of payments and transfers for current international transactions. Since January 1, 1999, the CFA franc has been pegged to the Euro at a fixed rate of $€ 1=$ CFAF 655.95. On April 30, 2007, the rate of the CFA franc in terms of the SDR was CFAF 733.8=SDR 1. Effective January 1, 2007, the exchange arrangement of the WAEMU countries has been reclassified to the category of conventional pegged arrangement from the category of exchange arrangement with no separate legal tender. The new classification is based on the behavior of the common currency, whereas the previous classification was based on the lack of a separate 
legal tender. The new classification thus reflects only a definitional change, and is not based on a judgment that there has been a substantive change in the exchange regime or other policies of the currency union or its members.

\section{Article IV Consultation}

Guinea-Bissau is on the 24-month consultation cycle. The last Article IV consultation discussions with Guinea-Bissau were held in Bissau January 12-26, 2010. The staff report (EBS/10/56) was discussed by the Executive Board on May 7, 2010.

\section{Technical Assistance}

\begin{tabular}{|c|c|c|c|}
\hline Department & $\begin{array}{l}\text { Type of } \\
\text { Assistance }\end{array}$ & Time of Delivery & Purpose \\
\hline MAE & Expert & $\begin{array}{l}\text { March } 20- \\
\text { July 23, } 1994\end{array}$ & Assisting in banking supervision \\
\hline MAE & Expert & $\begin{array}{l}\text { June } 6,1994- \\
\text { January } 26,1997\end{array}$ & Assisting in monetary policy \\
\hline MAE & Expert & $\begin{array}{l}\text { June-- } \\
\text { December } 1995\end{array}$ & Advising on bank accounting \\
\hline STA & Staff & March 4-15, 1996 & $\begin{array}{l}\text { Assessing the quality of monetary, balance of } \\
\text { payments, and government statistics }\end{array}$ \\
\hline FAD & Staff & $\begin{array}{l}\text { September 8- } \\
24,1996\end{array}$ & Advising on tax administration and tax policy \\
\hline FAD & Staff & March 9-25, 1997 & $\begin{array}{l}\text { Advising on import tariff reform and the design of a } \\
\text { general sales tax (GST) }\end{array}$ \\
\hline MAE & Expert & $\begin{array}{l}\text { June- } \\
\text { September } 1997 \\
\text { (three short-term } \\
\text { missions) }\end{array}$ & $\begin{array}{l}\text { Assisting in the transformation of the central bank } \\
\text { into a branch of the Central Bank of West African } \\
\text { States (BCEAO) }\end{array}$ \\
\hline FAD & Expert & $\begin{array}{l}\text { September 8- } \\
24,1997\end{array}$ & $\begin{array}{l}\text { Assisting in the design and implementation of GST } \\
\text { and in strengthening fiscal control }\end{array}$ \\
\hline FAD & Expert & $\begin{array}{l}\text { October } 19,1997- \\
\text { April } 10,1998\end{array}$ & Assisting in GST implementation \\
\hline FAD & Expert & June $3-15,1998$ & Assisting in GST implementation \\
\hline FAD & Expert & $\begin{array}{l}\text { January 1, 1999- } \\
\text { March 2, } 1999\end{array}$ & Tax administration advisor \\
\hline FAD & Expert & $\begin{array}{l}\text { April 15, 1999- } \\
\text { June 14, } 1999\end{array}$ & Tax administration advisor \\
\hline FAD & Staff & July 13-27, 1999 & Assessing budget management and the tax system \\
\hline FAD & Expert & $\begin{array}{l}\text { January 24, 2000- } \\
\text { March 5, } 2000\end{array}$ & Tax administration advisor \\
\hline FAD & Expert & January 2001 & Tax administration advisor \\
\hline FAD & Expert & February 2001 & Peripatetic public expenditure management advisor \\
\hline
\end{tabular}




\begin{tabular}{|c|c|c|c|}
\hline FAD & Expert & July 2001 & Peripatetic public expenditure management advisor \\
\hline FAD & Staff & April 2003 & Expenditure management \\
\hline FAD & Staff & April 2003 & Revenue administration \\
\hline STA & Expert & March 2003 & GDDS—Real sector statistics \\
\hline STA & Expert & May 2003 & GDDS_Fiscal statistics \\
\hline WEST AFRITAC & Resident Advisor & November 2003 & Public expenditure management \\
\hline WEST AFRITAC & $\begin{array}{l}\text { Resident Advisor; } \\
\text { Expert }\end{array}$ & February 2004 & Tax administration \\
\hline WEST AFRITAC & $\begin{array}{l}\text { Resident Advisor; } \\
\text { Expert }\end{array}$ & March 2004 & Customs administration \\
\hline WEST AFRITAC & Expert & May 2004 & Tax administration \\
\hline WEST AFRITAC & Resident Advisor & May 2004 & Public debt management \\
\hline WEST AFRITAC & Resident Advisor & June 2004 & Budget preparation and execution \\
\hline WEST AFRITAC & Resident Advisor & August 2004 & $\begin{array}{l}\text { Assessing the priority needs for technical assistance } \\
\text { and assisting the authorities with the preparation of } \\
\text { a work plan for improving compilation of balance } \\
\text { of payments and international investment position } \\
\text { statistics. }\end{array}$ \\
\hline WEST AFRITAC & $\begin{array}{l}\text { Resident Advisor; } \\
\text { Short-Term Expert }\end{array}$ & August 2004 & Government finance statistics \\
\hline WEST AFRITAC & Short-Term Expert & September 2004 & Government finance statistics \\
\hline WEST AFRITAC & Short-Term Expert & October 2004 & Customs administration \\
\hline WEST AFRITAC & Resident Advisor & November 2004 & Public expenditure management \\
\hline WEST AFRITAC & $\begin{array}{l}\text { Resident Advisor; } \\
\text { Short-Term Expert }\end{array}$ & November 2004 & Tax administration \\
\hline WEST AFRITAC & $\begin{array}{l}\text { Resident Advisor; } \\
\text { Short-Term Expert }\end{array}$ & January 2005 & Government finance statistics \\
\hline WEST AFRITAC & $\begin{array}{l}\text { Resident Advisor; } \\
\text { Short-Term Expert }\end{array}$ & February 2005 & Tax administration \\
\hline WEST AFRITAC & $\begin{array}{l}\text { Resident Advisor; } \\
\text { Short-Term Expert }\end{array}$ & March 2005 & Public expenditure management \\
\hline WEST AFRITAC & $\begin{array}{l}\text { Resident Advisor; } \\
\text { Short-Term Expert }\end{array}$ & April 2005 & Customs administration \\
\hline WEST AFRITAC & $\begin{array}{l}\text { Resident Advisor; } \\
\text { Short-Term Expert }\end{array}$ & May 2005 & Tax administration \\
\hline WEST AFRITAC & Resident Advisor & June 2005 & Microfinance supervision \\
\hline
\end{tabular}




\begin{tabular}{|c|c|c|c|}
\hline WEST AFRITAC & Resident Advisor & June 2005 & Microfinance supervision \\
\hline WEST AFRITAC & Resident Advisor & October 2005 & Government finance statistics \\
\hline WEST AFRITAC & $\begin{array}{l}\text { Resident Advisor; } \\
\text { Short-Term Expert }\end{array}$ & November 2005 & Customs administration \\
\hline WEST AFRITAC & $\begin{array}{l}\text { Resident Advisor; } \\
\text { Short-Term expert }\end{array}$ & November 2005 & Tax administration \\
\hline WEST AFRITAC & $\begin{array}{l}\text { Resident Advisor; } \\
\text { Short-Term Expert }\end{array}$ & November 2005 & Public expenditure management/accounting \\
\hline WEST AFRITAC & $\begin{array}{l}\text { Resident Advisor; } \\
\text { Short-Term Expert }\end{array}$ & January 2006 & Tax administration \\
\hline WEST AFRITAC & Short-Term Expert & March 2006 & Public expenditure management/accounting \\
\hline WEST AFRITAC & Resident Advisor & March 2006 & Real sector statistics \\
\hline WEST AFRITAC & Short-term expert & July 2006 & Government finance statistics \\
\hline WEST AFRITAC & Resident Advisor & July 2006 & Tax administration \\
\hline WEST AFRITAC & Short-term expert & July-August 2006 & Customs administration \\
\hline WEST AFRITAC & Resident Advisor & December 2006 & Public debt management \\
\hline WEST AFRITAC & Short-term expert & January 2007 & Government finance statistics \\
\hline WEST AFRITAC & Resident Advisor & January 2007 & Customs administration \\
\hline WEST AFRITAC & Resident Advisor & February 2007 & Tax administration \\
\hline WEST AFRITAC & Resident Advisor & March 2007 & Public finance management \\
\hline WEST AFRITAC & Short-term expert & June 2008 & Customs administration \\
\hline WEST AFRITAC & Short-term expert & June 2008 & Public Expenditure management \\
\hline STA & Expert & June 2008 & Balance of Payment Statistics \\
\hline WEST AFRITAC & Short-term expert & July 2008 & Government finance statistics \\
\hline WEST AFRITAC & Short-term expert & August 2008 & Multisector Statistics \\
\hline WEST AFRITAC & Short-term expert & September 2008 & Real sector Statistics \\
\hline
\end{tabular}




\begin{tabular}{|c|c|c|c|}
\hline WEST AFRITAC & Short-term expert & May 2009 & National Accounts \\
\hline WEST AFRITAC & Long-term expert & June 2009 & National Accounts \\
\hline WEST AFRITAC & Short-term expert & June 2009 & Public Expenditure management \\
\hline WEST AFRITAC & Short-term expert & June 2009 & Public Debt management \\
\hline WEST AFRITAC & Short-term expert & June 2009 & Bank Supervision \\
\hline WEST AFRITAC & Short-term expert & June 2009 & Public Finance management \\
\hline WEST AFRITAC & Short-term expert & September 2009 & Tax administration \\
\hline WEST AFRITAC & Short-term expert & September 2009 & Customs administration \\
\hline WEST AFRITAC & Short-term expert & November 2009 & Public Debt management \\
\hline WEST AFRITAC & Short-term expert & November 2009 & Real Sector Statistics \\
\hline West AFRITAC & Short-term expert & March 2010 & Government Finance Statistics \\
\hline FAD & Staff & September 2010 & Tax Revenue and Customs Administration \\
\hline West AFRITAC & Short-term expert & September 2010 & Expenditure management \\
\hline West AFRITAC & Short-term expert & September 2010 & National Accounts \\
\hline West AFRITAC & Short-term expert & February 2011 & Tax Administration \\
\hline West AFRITAC & Short-term expert & March 2011 & Government Finance Statistics \\
\hline West AFRITAC & Short-term expert & April 2011 & Public Debt Management \\
\hline West AFRITAC & Short-term expert & April 2011 & Public Expenditure Management \\
\hline
\end{tabular}

\section{Resident Representative}

The Resident Representative in Senegal also covered Guinea-Bissau from September 1997 through July 2007. The Resident Representative Office in Guinea-Bissau will be reopened as of June 2011. 


\begin{tabular}{|c|c|c|c|}
\hline \multicolumn{4}{|c|}{ Table 1: Guinea-Bissau-Arrangements with the IMF, 1984-2010 } \\
\hline Arrangement & Period & Amount Approved & Remarks \\
\hline $\begin{array}{l}\text { First credit tranche } \\
\text { purchase }\end{array}$ & August 27, 1984 & SDR1.875 million & \\
\hline $\begin{array}{l}\text { Structural Adjustment } \\
\text { Facility }\end{array}$ & $\begin{array}{l}\text { October 14, 1987- } \\
\text { October 13, } 1990\end{array}$ & SDR 5.25 million & $\begin{array}{l}\text { 2nd annual } \\
\text { arrangement delayed; } \\
\text { no } 3^{\text {rd }} \text { annual } \\
\text { arrangement. }\end{array}$ \\
\hline $\begin{array}{l}\text { Enhanced Structural } \\
\text { Adjustment Facility }\end{array}$ & $\begin{array}{l}\text { January 18, 1995-July } \\
24,1998\end{array}$ & SDR 10.5 million & $\begin{array}{l}\text { Arrangement increased } \\
\text { by SDR } 1.05 \text { million } \\
\text { (10 percent of quota) } \\
\text { with } 3^{\text {rd }} \text { annual } \\
\text { arrangement. }\end{array}$ \\
\hline $\begin{array}{l}\text { Emergency post- } \\
\text { conflict assistance }\end{array}$ & September 14, 1999 & SDR 2.13 million & \\
\hline $\begin{array}{l}\text { Emergency post- } \\
\text { conflict assistance }\end{array}$ & January 7,2000 & SDR 1.42 million & \\
\hline $\begin{array}{l}\text { Poverty Reduction and } \\
\text { Growth Facility }\end{array}$ & $\begin{array}{l}\text { December 15, 2000- } \\
\text { December 14, } 2003 \\
\end{array}$ & SDR 14.2 million & $\begin{array}{l}\text { PRGF expired without } \\
\text { completion of a review. }\end{array}$ \\
\hline $\begin{array}{l}\text { Emergency post- } \\
\text { conflict assistance }\end{array}$ & January 10, 2008 & SDR 1.77 million & \\
\hline $\begin{array}{l}\text { Emergency post- } \\
\text { conflict assistance }\end{array}$ & May 20, 2009 & SDR 1.77 million & \\
\hline $\begin{array}{l}\text { Extended Credit } \\
\text { Facility }\end{array}$ & May 7, 2010 & SDR 22.365 million & \\
\hline
\end{tabular}

Source: International Monetary Fund. 


\section{Guinea-Bissau: Relations with the World Bank Group}

\section{As of April 10, 2011}

1. As of April 10, 2011, IDA had approved altogether 33 credits and grants for GuineaBissau for a total value of commitments of US\$362.8 million equivalent. The most important sectors, so far, have been the following: Transport/Infrastructure/Energy (eight operations, total commitments: US\$121.5 million), Structural Adjustment/Development Policy Operations (budget support, three operations approved in 1987-2000, and three since 2008, of which one exclusively for the payment of primary teachers' salaries; total commitments: US\$87.4 million) and Social Sectors, including Health and Education (eight operations and US\$ 55.5 million of total commitments). Two operations were implemented to support private sector development (overall commitment: US\$ 41million), and three to provide technical assistance for economic management (overall commitment: US\$22.9 million). Two IDA-funded credits supported the extractive industry sector, i.e. petrol exploration, in the 1980s with total commitments of US\$19.9 million, and four IDA-funded projects have been supporting agriculture/natural resources management (including one new regional Fisheries Project), so far, with total commitments of 14.6 million, respectively, for this area of intervention.

\section{Budget Support Operations}

2. Since 2008, (after a period of discontinuation of budget support due to political instability), the Bank initiated new budget support operations to Guinea-Bissau, first with an emergency economic rehabilitation operation approved in 2008 that provided funds exclusively for the payment of primary teachers' salaries, and then with a series of programmatic Development Policy Grants, of which the first one was approved in June 2009 and disbursed in early September (US\$8 million), the second one approved in June 2010 and disbursed in December 2010 (US\$6 million), and a third one currently under preparation for Board Approval in June 2011 (US\$6 million).

\section{Lending program}

3. In June 2009, the Bank approved an Interim Strategy Note for Guinea-Bissau for 2009-2011, based on two pillars: (i) Strengthening Economic Management and Laying the Foundations for Improvement in the Productive Sectors; and (ii) Increased Access to Basic Services. Capacity Development for efficient governance and project implementation is a cross-cutting topic. A new Country Assistance Strategy (CAS) is envisaged to be delivered in the second semester of 2011.

4. The current IDA Portfolio is composed of five active operations with a total commitment of US\$40.6 million, including a Multi-Sector Infrastructure Rehabilitation Project (US\$15 million) supplemented by a new Emergency Electricity \& Water Rehabilitation Project (US\$12.7), a Community-Driven Development Project (US\$5 million), a new Biodiversity Conservation Project (US\$1.9 million) and the Guinea- 
Bissau component of a regional West Africa Fisheries Program (US\$6 million) As of April 10, 2011, the IDA undisbursed balance was US\$39.79 million.

5. The currently active Trust Fund portfolio implemented by the World Bank amounts to a total sum of about US\$18 million. It includes an Emergency Food Security Support operation funded through the Food Price Crisis Response Trust Fund with contributions by the EU. One component of this project (school feeding, food for work for rural infrastructure) is implemented by the World Food Program. Further, the IDA-funded Community-Driven Development operation is complemented by a similar Participatory Rural Development operation of US\$5 million, funded from the State and Peace Building (SPF) Trust Fund window for fragile states. The SPF also provides funding for Technical Assistance to the Ministry of Finance on Economic Management, complementing the IDA budget support, and an operation to support the implementation of the National Health Development Plan. Further complementary (co-) funding is provided by the Global Environment Facility (GEF) to the Biodiversity Conservation Project. An important multi-donor Education sector operation is currently under preparation in close cooperation with other donors, i.e. UNICEF, and has been envisaged to be submitted to the Education for All-Fast-Track Initiative (EFAFTI) in May 2011; however, due to less than expected resource allocation of the EFA-FTI Catalytic Fund, the approval of the operation might be delayed for several months.

\section{Non-lending Program}

6. On December 14, 2010, the IDA Board of Executive Directors approved the achievement of the HIPC Completion Point for Guinea-Bissau. IDA provided MDRI debt forgiveness by irrevocably canceling Guinea-Bissau's debt service obligations for credits disbursed before December 31, 2003, and still outstanding at December 31, 2010, after the application of HIPC assistance and topping up. In March 21, 2011, the IDA Board of Executive Directors approved the topping-up additional debt relief. IDA debt service reduction under the enhanced HIPC initiative, MDRI and topping-up assistance at Completion Point amounts US\$263.7 million.

7. At current the Bank team is finalizing work on a Country Economic Memorandum which will identify key drivers for transformative growth in preparation of the upcoming Country Assistance Strategy for the period of 2011-2014. The team is also preparing a Capacity Development Action Plan to be annexed to the CAS. Technical assistance is currently provided in the area of Economic Governance, and prepared for Extractive Industries Value Chain Management in view of the government's request to join the EITI. Further, IDA is preparing technical assistance for the establishment of a Global Development Learning Center for which funding options are being explored with the government of Brazil or possible other donors.

8. The World Bank Institute (WBI) envisages to launch more activities in the coming fiscal year, through inputs and capacity development activities in areas such as leadership 
and governance (demand-side governance), and possibly by contributing to the provision of learning content through the Global Development Learning Center in Bissau.

\section{IFC and MIGA:}

9. IFC: An IFC exploration mission visited Guinea-Bissau in November 2010, and identified several areas for potential future intervention, in particular, the electricity and cashew sectors, as well as improvement of financial markets and investment climate.

10. MIGA's portfolio in Guinea-Bissau consists of two projects, sponsored by Senegalese and Malian investors. The investments, in support of the country's telecommunications and tourism sectors, have a combined gross exposure of $\$ 17.1$ million. The Agency has not received any formal requests to provide guarantees for investments in Guinea-Bissau during FY11. 


\section{Guinea-Bissau: Relations with the African Development Bank Group}

\section{As of March 2011}

1. By March 2011, the AfDB had approved 38 operations for Guinea-Bissau, excluding multinational projects. These include 24 projects, 3 studies and 7 institutional supports, 3 special relief fund operations and 1 line of credit representing a net commitment of UA 188.08 millions. $31.2 \%$ of these operations have been in the social sector, $21 \%$ in the multisector, $19.4 \%$ in agriculture/fisheries, $18.4 \%$ in transport and 10\% in infrastructure equipment. A total of UA 155.96 millions representing $82.9 \%$ have been disbursed. As of March 2011, the active portfolio comprises five ongoing projects representing a total net amount of UA 44.75 million and a disbursed amount of UA 19 million.

\section{Structural adjustment credits}

2. AfDB has approved a total of UA 13 million for structural adjustment operations. These include two structural adjustment credits (SACs), one supplementary SAC, and one economic rehabilitation and recovery credit (ERRC). The SACs supported the government's program in the areas of economic liberalization and reform of the public administration and public enterprise sectors and the ERRC supports peace building following the political unrest, promotes the revival of the economy and encourages the pursuit of reforms.

\section{Lending Program}

3. During the period January 2008-March 2011, AfDB approved an interim HIPC debt release (US\$17.48 million, a Fragile State Facility grant (UA2 million), a fish sector support grant (UA2 million), a health sector grant (UA6 million), an emergency support grant to cholera (UA0.33 million), a capacity building grant to public administration (UA7.86 million) and an emergency budget support to budgetary reforms (UA5.7 million). The Bank will be preparing this year another Budget Support (UA 9 million) and a capacity building project (UA 3.5 million) expected to be approved this year.

\section{Nonlending Program}

4. The Bank undertook a portfolio review in 2008, a mid-term review of its Country Strategy Paper (CSP) in 2009 as well as a combined portfolio review and completion report of its CSP 2005-2010, in October 2010. The Bank also participated in a Public Expenditure Management and Financial Accountability Review (PEMFAR) in 2009 in collaboration with the World Bank and the European Commission. The Bank is preparing in 2011 a new CSP 2011-2015. 


\section{Guinea Bissau: Statistical Issues}

As of April 11, 2011

\section{Assessment of Data Adequacy for Surveillance}

General: Data have serious shortcomings that significantly hamper surveillance. Data compilation was impaired during the 1998-99 civil conflict. The authorities have improved their data compilation in the recent years, with technical assistance from international and regional institutions.

National Accounts: Guinea-Bissau made progress on national account statistics in 2009 . The National Institute of Statistics and Censuses (INEC) published in the second quarter of 2010 revised national account data for 2003-08, based on the $S N A 93$. The new data have a broader coverage of all sectors of the economy, and as a result the GDP level has doubled. The new data covers GDP in both constant and current prices, and GDP deflators.

Price statistics: Since July 2002, a harmonized consumer price index (CPI) has been compiled, based on the same structure as in other West African Economic and Monetary Union (WAEMU) countries. Price data are collected only for the capital city, Bissau.

Government finance statistics: Government finance statistics missions contributed to: (1) training on the methodology for Guinean officials, (2) implementation of a budgetary data collection program, and (3) setting up a source database and a worksheet table for the State Financial Operations (TOFE). Since 2007, the monthly TOFE is compiled on a regular basis and used as a basic tool in monitoring the program with the IMF. In March 2010, a government finance statistics (GFS) mission from West AFRITAC provided technical assistance to the authorities in compiling and disseminating GFS and implement an action plan designed to improve GFS data dissemination to users.

Monetary statistics: Monetary and financial statistics, compiled and disseminated by the regional Central Bank of West African States (BCEAO), are broadly adequate and their institutional coverage is comprehensive. Accuracy is somewhat hampered by the use of outdated sorting coefficients to estimate cross border amounts of banknotes among BCEAO countries, which in turn are used to estimate currency in circulation and to adjust the net foreign assets of each member country. 
Balance of Payments Statistics: Guinea-Bissau reports trade data to AFR for operational purposes, using information from customs. BOP data are weak, mostly due to substantial unregistered trade and inconsistencies between data on net foreign assets as reported by the BCEAO and other economic indicators. In April 2008, an STA TA mission assessed the methodology and compilation procedures for the production of BOP statistics and provided training to BCEAO staff. The mission found that the large number of small-scale operators, a large informal sector, and institutional weaknesses (only some 20 percent of businesses file tax returns) hamper the data collection.

While no external debt data are published by the Ministry of Finance, stock and flow data are regularly produced and transmitted to the BCEAO. The outstanding stock of debt comprises only about 200 active loans.

\section{Data Standards and Quality}

Guinea-Bissau participates in the GDDS No data ROSC is available. since November 2001. Metadata for all data categories and plans for improvement need to be updated.

\section{Reporting to STA}

There is currently no monthly, quarterly or annual government finance data submitted for reporting in the International Financial Statistics (IFS) or the Government Finance Statistics Yearbook. Monthly data on monetary statistics for Guinea Bissau are reported on a regular basis for publication in the IFS, albeit with some delays. Guinea Bissau reports balance of payments (BOP) and international investment position statistics to STA on an annual basis, albeit with considerable delays. 


\section{Guinea-Bissau: Table of Common Indicators Required for Surveillance}

(As of April 20, 2011)

\begin{tabular}{|c|c|c|c|c|c|}
\hline & $\begin{array}{c}\text { Date of latest } \\
\text { observation }\end{array}$ & Date received & $\begin{array}{c}\text { Frequency } \\
\text { of } \\
\text { data }^{7}\end{array}$ & $\begin{array}{l}\text { Frequency } \\
\quad \text { of } \\
\text { reporting }^{7}\end{array}$ & $\begin{array}{l}\text { Frequency } \\
\text { of } \\
\text { publication }^{7}\end{array}$ \\
\hline Exchange rates & Current & Current & $\mathrm{D}$ & $\mathrm{D}$ & $\mathrm{D}$ \\
\hline $\begin{array}{l}\text { International reserve assets and reserve liabilities of } \\
\text { the monetary authorities } 1\end{array}$ & Nov. 2010 & Mar. 2011 & M & M & M \\
\hline Reserve/base money & Nov. 2010 & Mar. 2011 & M & M & M \\
\hline Broad money & Nov. 2010 & Mar. 2011 & M & M & M \\
\hline Central bank balance sheet & Nov. 2010 & Mar. 2011 & M & M & M \\
\hline Consolidated balance sheet of the banking system & Nov. 2010 & Mar. 2011 & M & M & M \\
\hline Interest rates $^{2}$ & Nov. 2010 & Mar. 2011 & M & M & M \\
\hline Consumer price index & Feb. 2010 & Mar. 2011 & M & M & M \\
\hline \multicolumn{6}{|l|}{$\begin{array}{l}\text { Revenue, expenditure, balance and composition of } \\
\text { financing }^{3} \text { - general government }\end{array}$} \\
\hline $\begin{array}{l}\text { Revenue, expenditure, balance and composition of } \\
\text { financing }{ }^{3} \text { - central government }\end{array}$ & Dec. 2010 & Mar. 2011 & M & Q & Q \\
\hline \multicolumn{6}{|l|}{$\begin{array}{l}\text { Stocks of central government and central } \\
\text { government-guaranteed debt }\end{array}$} \\
\hline External current account balance & 2009 & Mar. 2011 & A & I & I \\
\hline Exports and imports of goods and services & 2009 & Mar. 2011 & A & I & I \\
\hline GDP/GNP & 2009 & Forthcoming & A & I & I \\
\hline \multicolumn{6}{|l|}{ Gross external debt } \\
\hline International Investment Position ${ }^{6}$ & 2009 & Mar. 2011 & A & & \\
\hline
\end{tabular}

${ }^{1}$ Any reserve assets that are pledged of otherwise encumbered should be specified separately. Also, data should comprise shortterm liabilities linked to a foreign currency but settled by other means as well as the notional values of financial derivatives to pay and to receive foreign currency, including those linked to a foreign currency but settled by other means.

${ }^{2}$ Both market-based and officially determined, including discount rates, money market rates, rates on treasury bills, notes and bonds.

${ }^{3}$ Foreign, domestic bank, and domestic nonbank financing.

${ }^{4}$ The general government consists of the central government (budgetary funds, extra budgetary funds, and social security funds) and state and local governments.

${ }^{5}$ Including currency and maturity composition.

${ }^{6}$ Includes external gross financial asset and liability positions vis-à-vis nonresidents.

${ }^{7}$ Daily (D), Weekly (W), Monthly (M), Quarterly (Q), Annually (A), Irregular (I); Not Available (NA). 
Press Release No. 11/193

International Monetary Fund

FOR IMMEDIATE RELEASE

Washington, D.C. 20431 USA

May 24, 2011

\section{IMF Executive Board Completes Second Review Under the ECF Arrangement with Guinea-Bissau and Approves US\$3.85 Million Disbursement}

The Executive Board of the International Monetary Fund (IMF) has completed the second review of Guinea-Bissau's economic performance under a three-year Extended Credit Facility (ECF) arrangement. ${ }^{1}$ The Board's decision, which was taken on a lapse-of-time basis, ${ }^{2}$ enables the authorities to draw an additional SDR 2.414 million (about US\$3.85 million), bringing total disbursements under the arrangement to an amount equivalent to SDR 12.709 million (about US\$20.27 million).

Satisfactory policy implementation continues in the Fund-supported program under challenging conditions. The authorities have met all performance criteria through endDecember 2010 and all structural reforms for the second review.

Sound macroeconomic policies, strengthened institutions, and debt relief have stabilized the economy and supported confidence building. While medium-term growth prospects are bright, huge developmental challenges remain. It is critical that the government maintain the reform momentum and continue to build on the satisfactory performance under the ECF.

The three-year ECF arrangement for Guinea-Bissau was approved on May 7, 2010 (see Press Release No. 10/185) in an amount equivalent to SDR 22.365 million (about US\$33.3 million, or 157.5 percent of the country's quota in the Fund). On December 16, 2010 the Executive Boards of the IMF and the World Bank's International Development Association decided to support US\$1.2 billion in debt relief for Guinea-Bissau under the Heavily Indebted Poor Countries (HIPC) Enhanced Initiative and the Multilateral Debt Relief Initiative (MDRI—see Press Release No. 10/498).

\footnotetext{
${ }^{1}$ The Extended Credit Facility (ECF) has replaced the Poverty Reduction and Growth Facility (PRGF) as the Fund's main tool for medium-term financial support to low-income countries by providing a higher level of access to financing, more concessional terms, enhanced flexibility in program design features, and more focused streamlined conditionality. Financing under the ECF carries a zero interest rate, with a grace period of 51/2 years, and a final maturity of 10 years (http://www.imf.org/external/np/exr/facts/ecf.htm). The Fund reviews the level of interest rates for all concessional facilities every two years.

2 The Executive Board takes decisions under its lapse-of-time procedure when it is agreed by the Board that a proposal can be considered without convening formal discussions.
}

Washington, D.C. 20431 • Telephone 202-623-7100 • Fax 202-623-6772 • www.imf.org 